\title{
Erfolgsfaktoren für die Kundengewinnung mit digitalem Marketing
}

\section{Am Beispiel des Online Marketing Tools Google AdWords}

\author{
Seraina Ulber und Etienne Rumo
}

\begin{abstract}
Zusammenfassung
Den Absatz zu steigern, ist das übergeordnete Ziel von effizientem Marketing. Die Digitalisierung begründet die Umsetzung vieler Online-Marketingaktivitäten. Ein wichtiger Player im digitalen Marketing ist Google AdWords, ein Tool, welches es Unternehmen ermöglicht, dass deren Produkte und Dienstleistungen von potenziellen Kunden gefunden werden, wenn auf der Suchmaschine von Google danach gesucht wird. Die Identifikation von Erfolgsfaktoren bei der Arbeit mit Google AdWords ist aufgrund der Schnelllebigkeit des Themenfelds sowie des komplexen AdWords-Auktionssystems eine Herausforderung. Eine Fallstudienanalyse, in deren Rahmen erfahrene Unternehmungen beim Marketing mit Google AdWords sowie Fachexperten befragt werden, deckt die Erfolgsfaktoren für Marketingmaßnahmen mit Google AdWords auf. Eine moderne und nutzerfreundliche Webseite mit relevanten Inhalten sowie eine hohe Relevanz zwischen Keyword, Werbeanzeige und Zielseite sind essenzielle Voraussetzungen für erfolgreiche Google-AdWords-Kampagnen.
\end{abstract}

S. Ulber $(\bowtie)$

Chur, Schweiz

E-Mail: seraina.ulber@bluemail.ch

E. Rumo

Berner Fachhochschule Wirtschaft, Bern, Schweiz

E-Mail: etienne.rumo@bfh.ch 


\subsection{Einleitung}

Nachdem Edmund Jerome McCarthy (1960) als Erster auf die vier Hauptachsen des Marketings einging und die Verflechtung dieser Komponenten den Marketing-Mix nannte, welcher die weltbekannten 4 P's Product (Produkt), Place (Vertrieb), Promotion (Kommunikation) und Price (Preis) beinhaltet und seit jeher die Basis von jeder Marketingstrategie stellt, wurden die vier P's bald auch im digitalen Marketing unentbehrlich. Der Megatrend rund um die Digitalisierung und damit einhergehend die Erfindung des World Wide Web im Jahr 1989 haben die Anforderungen an Marketingstrategien nachhaltig geprägt. Der Einbezug von Online-Marketing stellt heute einen gleichwertigen oder sogar überwiegenden Aspekt der Marketingmaßnahmen von Unternehmungen dar. Beim Online-Marketing werden die Planung sowie die Umsetzung von Marketingaktivitäten unter Verwendung von digitalen Systemen beschrieben (Wendeler o. J.). Ein bedeutender Player im Zeitalter der Digitalisierung ist die Unternehmung Google LLC, welche im Jahr 2000 den Ableger Google AdWords lanciert hat. Das Tool beschäftigt sich damit, Usern, die mit der Suchmaschine von Google nach etwas Bestimmtem suchen, zielgerichtete und relevante Werbung zu schalten (Weller und Calcott 2012). „Reach your customers in the moment that matters with the relevant information“ (Leu 2018a), lautet der Slogan, den Google im Rahmen der Vermarktung seiner AdWords verwendet. Google ist bestrebt, sich ständig weiterzuentwickeln, damit sie ihrem Credo gerecht wird und am Puls der Zeit ist. Die Suchmaschine von Google ist weltweit die mit großem Abstand Bedeutendste. Google hatte im Jahr 2017 einen Marktanteil von über $92 \%$ inne, während der erste Verfolger Bing einen Marktanteil von weniger als $3 \%$ aufwies (Lunapark 2017). Zudem bleibt Google der Spitzenreiter bei der Frage, wer den größten Anteil am globalen digitalen Werbemarkt besitzt. Der Suchmaschinengigant liegt mit einem geschätzten Anteil von 31,2 \% im Jahr 2019 deutlich vorne (Internet World Business 2018).

Für die erfolgreiche und effiziente Arbeit mit Google AdWords sind die Erfolgsfaktoren von Unternehmung zu Unternehmung verschieden und stark von der Sortimentsvielfalt und der Branche abhängig. Die Identifikation von Erfolgsfaktoren für das Marketing mit Google AdWords stellt sich daher als sehr vielfältig und herausfordernd heraus. Trotzdem gibt es grundlegende, allgemeingültige Faktoren, welche berücksichtigt werden sollten, um mit Google AdWords erfolgreiche Marketingmaßnahmen zu implementieren. Eine weitere Challenge des Marketings mit Google AdWords besteht darin, der sich ständig ändernden Dynamik des digitalen Marketings gerecht zu werden. Was heute allgemeingültig scheint, kann bereits morgen veraltet sein. Die Funktionsweise von Marketing mit Google AdWords steht in starker Verbundenheit mit dem Google Algorithmus, der darüber entscheidet, welche Anzeige von welchem Anbieter wann ausgespielt wird. Aufgrund der Tatsache, dass der Google-Algorithmus ein einmaliges Phänomen in Sachen Komplexität und Genialität ist und im Hintergrund Auktionen zur Ausspielung 
jeder einzelnen Werbeanzeige durchführt, ist das Marketing mit Google AdWords teilweise unberechenbar (VCS 1 2018; VCS 2 2018; VCS 4 2018; VCS 5 2018).

Der Output dieses Beitrags deckt Erfolgsfaktoren des Marketings mit Google AdWords im Rahmen einer empirischen Untersuchung auf. Dabei wird eine Art Anleitung für erfolgreiche Google-AdWords-Strategien aufgezeigt. Die Identifikation der Erfolgsfaktoren beim Marketing mit Google AdWords dient als Ausgangslage für Unternehmungen, welche in der Digitalisierung einen Schritt vorankommen möchten und in Zukunft anhand von Google AdWords Werbung platzieren oder deren Skills zur Optimierung von bestehenden AdWords-Kampagnen verbessern möchten.

\subsection{Online-Marketing}

\subsubsection{Bedeutung und Einordnung}

Die Planung, Koordination und Kontrolle der auf die relevanten Märkte ausgerichteten Aktivitäten einer Firma zur Erreichung der Unternehmensziele werden als Marketing beschrieben. Dazu gehören auch absatzorientierte Instrumente wie Werbung, wobei die funktionale Perspektive von Marketing weit darüber hinausgeht. Die Bedürfnisse der adressierten Akteure und letztendlich deren Befriedigung sind ebenso Teil des Marketings. Direkte Verkaufsförderung oder die Steigerung der Bekanntschaft von Marken sind weitere entscheidende Faktoren im Marketingprozess. Online-Marketing im Speziellen ermöglicht komplett neue Marketingalternativen und stellt nicht nur einen weiteren Kanal für die Umsetzung der klassischen Produkt-, Preis-, Distributions- und Kommunikationsstrategien dar, sondern kann als grundlegender Innovationsprozess verstanden werden (Griesbaum 2012).

Die datengetriebene Automatisierung des Marketings ist seit Jahren weltweit ein nachhaltiges und stets aktuelles Thema. Die Rede ist dabei vom digitalen bzw. OnlineMarketing. Eine sehr pragmatische Definition dieser Art von Marketing besagt, dass es die Umsetzung des Marketings in Online-Medien bedeutet und der Fokus häufig auf der Kommunikationspolitik (Promotion) liegt (Busch 2014). Das Online-Marketing ist ein wichtiger Bestandteil des Digital Business und für die heutige Unternehmensführung ein unentbehrlicher Player. Es fasst alle Marketingaktivitäten zusammen, die auf der Grundlage von internetbasierten Technologien durchgeführt werden (Griesbaum 2012). In den letzten zwei Jahrzehnten hat sich die digitale Welt rasant entwickelt und ist heute für einen Großteil der Konsumenten nicht mehr aus dem Alltag wegzudenken (Dänzler und Heun 2014). Die weltweiten Ausgaben für Werbung im Internet überstiegen im Jahr 2017 gemäß Herrmann (2017) die Grenze von 200 Mrd. US\$. Eine zentrale Rolle in diesem Umfeld hat die Unternehmung Google LLC inne. Google ist die meist besuchte Webseite der Welt, Marktführer bei weltweiten Suchanfragen und somit ein prägender Player des digitalen bzw. Online-Marketings (Weller und Calcott 2012). 
Bereits um die Jahrtausendwende und somit nach gut 10 Jahren Existenz des Internets wurde deutlich, dass die bestehenden Marktstrukturen tiefgreifende Änderungen durchgehen und sich dies in Zukunft weiter verstärken wird. Link (2000) hielt zudem fest, dass das Online-Marketing essenzielle Weiterentwicklungen mit sich bringen wird, die für das Offline-Marketing nicht relevant waren. Dabei handelt es sich beispielsweise um Sicherheitstechnologien und damit einhergehend um die Internetkriminalität, welche eng verknüpft ist mit der Datenschutzproblematik. Eine Studie hat ergeben, dass für mehr als $50 \%$ der deutschen Bevölkerung eine steigende Digitalisierung dazu führt, dass deren Angst vor Internetkriminalität ebenfalls steigt (Hamburg 2017). Das Misstrauen, das Skeptiker dem Internetmedium entgegenbringen, basiert gemäß Frost (2013) auf der Sorge um die eigenen Daten und anderen Sicherheitsbedenken. Obschon das World Wide Web gemäß BFS (2017) von $79 \%$ der europäischen Bevölkerung und $88 \%$ der schweizerischen Bevölkerung wöchentlich genutzt wird und der Anteil an Internet-Nutzern somit immens groß ist, bestehen Hürden für die Nutzung. Die Datenschutzproblematik ist zweifelslos eine dieser Hürden.

Das Europäische Parlament (2018) hat festgehalten, dass $37 \%$ der Konsumenten fast ausschließlich online shoppen. Zum anderen ist das Internet für $57 \%$ der Konsumenten die erste Quelle, um sich vor dem Entscheid über den Kauf oder eines Produkts zu informieren (Hohlstein 2016). Ersteres Statement deutet den anhaltenden Wandel in Richtung Online-Shopping an, während letztere Aussage aufzeigt, wie wichtig es für Unternehmungen ist, mit den eigenen Produkten und/oder Dienstleistungen online im World Wide Web präsent zu sein. Der Trend der Digitalisierung und damit derjenige des Wandels vom Offline- hin zum Online-Marketing schlägt sich unter anderem bei den Werbeausgaben durch. Während die Prozentzahlen der globalen Werbeausgaben für die Medien Zeitungen, Magazine, TV und Radio seit 2012 stets sinken, erhöhten sich die digitalen, prozentualen Werbeausgaben von 18,3\% im Jahr 2012 zu geschätzten $27 \%$ im Jahr 2019 (Stone 2014; Herrmann 2017).

\subsubsection{Echtzeit- und crossmediales Marketing}

Ein wichtiger Ableger des Online-Marketings ist das Realtime-Advertising-Prinzip, welches den Verkauf von Online-Werbeplätzen in Echtzeit beschreibt. Im Gegensatz zum traditionellen Display-Markt, in dem Werbeplätze für eine bestimmte Zeitspanne fest erworben werden, werden diese beim Realtime Advertising in Echtzeit versteigert und ausschließlich für passende Nutzer angezeigt (Advidera o. J.). Zusammengefasst dreht sich alles um die intensivere Nutzung von Daten, Technik und künstlicher Intelligenz im Marketing, mit dem übergeordneten Ziel einer Verbesserung der Marketingeffizienz in Echtzeit (Busch 2014). Die zunehmende Digitalisierung ermöglicht unmittelbare Feedbacks und ungeahnte Optimierungsmöglichkeiten. Werbung kann exakt passend gesteuert, Motive und Botschaften abhängig vom Individuum und der Situation augenblicklich angepasst werden. Ressourcenintensive Arbeiten wie die Mediaplanung nach 
Umfeld sowie psychografische Zielgruppenmodelle erübrigen sich, denn das Marketing kann Individuen erkennen, diese ansprechen und den Erfolg sofort messen. Diese Vorteile bringt das Echtzeitmarketing, das passend mit den Begriffen Geschwindigkeit, Reiz und Reaktion beschrieben werden kann (Engel 2015). Obschon das Echtzeitmarketing einige grundlegende Prinzipien des Marketings über den Haufen wirft, hält Busch (2014) fest, dass es sich beim Realtime Advertising nicht um einen plötzlich aufgetretenen Hype handelt, sondern sich dieses über einen Zeitraum von etwas mehr als fünf Jahren entwickelte. Dabei waren verschiedene Entwicklungsstränge ausschlaggebend, welche im Endeffekt und in Kombination eine hohe Relevanz erfahren haben. Diese Entwicklungen stellen zusammen die Möglichkeiten dar, welche die Marketingbranche derzeit in der Praxis bei der Lösung von Problemstellungen exploriert. Das Interesse des Werbemarkts für derartige Technologie und Wissenschaft setzt enorme finanzielle Mittel frei, die eine zusätzliche Beschleunigung des bisherigen Weges ermöglichen. Realtime Advertising ist ein neues, wertschöpfendes Prinzip im Umgang mit digitaler Werbung, in welchem der unaufhaltsame Prozess der Digitalisierung von Werbung und Marketing stets in Betracht gezogen werden muss.

Im Zuge der rasanten Entwicklung des Online-Marketings darf nicht vergessen werden, dass die Wichtigkeit des Offline-Marketings weiterhin bestehen bleibt. Die Kombination der Marketingzweige online und offline wird crossmediales oder auch integriertes Marketing genannt und stellt für Unternehmungen ein hochspannendes Tool dar, um sich Wettbewerbsvorteile zu verschaffen, wenn die Integration und Verflechtung beider Zweige erfolgreich gestaltet werden. Der Prozess des crossmedialen Marketings bezeichnet gemäß der Autorin Stich (2017) die Verknüpfung der Online-Werbemaßnahmen mit der Offline-Werbung. Das crossmediale Marketing wird hauptsächlich der Kommunikationspolitik zugeordnet. Die integrierte Marketingkommunikation beabsichtigt einen abgestimmten Einsatz der unterschiedlichen Kommunikationsinstrumente, damit schlüssige, und überzeugende Aussagen über Produkte, Dienstleistungen und Unternehmen kommuniziert werden können. Dabei muss ersichtlich sein, dass anhand von verschiedenen Tools identische Aussagen verbreitet werden. Diese Messages werden parallel zu und schlüssig mit den verschiedenen Kommunikationsinstrumenten in einer ganz bestimmten Zeitperiode gestreut (Rumo 2017).

\subsection{Google AdWords}

Google AdWords ist ein im Oktober 2000 lanciertes Programm der Firma Google LLC, welches aus dem Online-Marketing-Mix nicht mehr wegzudenken ist. Es handelt sind dabei um die Werbeanzeigen auf den Ergebnisseiten von Google. Gemäß Google AdWords (o. J.) soll es für Unternehmungen möglich sein, von potenziellen Kunden immer dann gefunden zu werden, wenn bei Google gezielt nach Produkten oder Dienstleistungen gesucht wird. Google AdWords ist jedoch nicht nur für die werbenden Händler interessant und vielversprechend, sondern auch für die Gründerfirma lukrativ. 
Das AdWords-Programm hat das gesamte Unternehmen verändert und Google endlich eine richtige Einnahmequelle beschert. Seit der Einführung des Tools ist AdWords die Haupteinnahmequelle des Unternehmens und auch heute noch für fast $90 \%$ des Umsatzes verantwortlich (GoogleWatchBlog 2015).

Ein Erfolgsfaktor für die Allgegenwärtigkeit von Google AdWords im OnlineMarketing ist deren Kostenmodell, Pay per Click genannt. Dieses ist so strukturiert, dass Werbende nicht für Einblendungen der Anzeigen, sondern lediglich für Klicks auf die Werbeanzeigen bezahlen. Google AdWords stellt somit eine der effektivsten Werbemöglichkeiten im Internet dar und ist weltweit die bekannteste Werbealternative (Google AdWords o. J.; Michnew 2015). Bis zu 90 \% der Konsumenten, welche eine Kaufabsicht haben, recherchieren vor dem Kauf in Suchmaschinen (DIM Deutsches Institut für Marketing GmbH o. J.). Gemäß Beck (2011) werden weltweit täglich 3,6 Mrd. Suchanfragen auf Google gestellt. Diese Fakten beweisen, dass es sich heutzutage kaum ein Unternehmen leisten kann, auf die Präsenz auf Suchmaschinen zu verzichten. Bei jeder Suchanfrage haben Unternehmungen jeglicher Branchen die Möglichkeit, sich selbst und ihre Webseite zu präsentieren, damit Neukunden anzuwerben und Umsatz zu generieren. Studers (2008) Google-AdWords-Studie hat überdies ergeben, dass Unternehmungen dank Google AdWords nicht lediglich den Absatz fördern und Leads generieren können, sondern auch, dass die bloße Einblendung von AdWords-Anzeigen ebenfalls einen deutlich nachweisbaren Branding-Effekt hat. Dieser äußerst begehrte Effekt wurde Google AdWords lange Zeit abgesprochen, konnte durch die Google-Studie „Markenbildung mit AdWords“ aber bestätigt werden. Eine Marke steigert seine Bekanntheit dank Google AdWords im Schnitt um $21 \%$ (Busch 2014).

Der Nutzen von Google AdWords lässt sich dadurch bestätigen, dass Menschen sich online über ein Produkt oder eine Dienstleistung informieren, für das bzw. die sie sich interessieren. Suchmaschinen sind dafür das meistgenutzte Medium. Aufgrund dessen kann festgehalten werden, dass die Suchmaschinenwerbung eine enorm hohe Reichweite hat. Da die Zielgruppe den Werber erst bei konkretem Bedarf sucht, findet und kontaktiert, lässt sich die Höhe an Einblendungen der Anzeigen sehr gezielt steuern, wenn strategisch gute Keywords eingebucht werden. Da die Suchmaschinenwerbung den Nutzer im entscheidenden Moment erreicht - eben erst und nur dann, wenn der User aktiv danach sucht - ist sie äußerst relevant und der generierte Traffic auf der Webseite des Werbenden sehr hochwertig (Yapi 2016).

\subsubsection{AdWords-Grundlagen}

Im Gegensatz zur Suchmaschinenoptimierung (Search Engine Optimization SEO), bei welcher technische und inhaltliche Maßnahmen ergriffen werden, damit die eigene Webseite möglichst weit oben in den natürlichen Suchergebnissen positioniert werden kann, geht es bei der Suchmaschinenwerbung (Search Engine Advertising SEA) um kostenpflichtige Textanzeigen, die aufgrund eines gewählten Keywords geschaltet werden. 
Aufgrund von zwei unterschiedlichen, voneinander unabhängigen Systemen erfolgt die Schaltung der natürlichen bzw. der bezahlten Anzeigen. Die beiden Systeme beeinflussen einander nicht (Yapi 2016).

Der grundsätzliche Ablauf von Google AdWords ist denkbar simpel und pragmatisch zu erklären. Der erste Schritt, welcher Google AdWords ins Spiel bringen, ist die Suchanfrage eines Nutzers auf der Google Webseite. Als Ergebnis erhält der User verschiedene Vorschläge, die organischer (SEO) sowie auch bezahlter (SEA) Natur sind. Bezahlte Suchergebnisse sind oben links unter dem Titel mit „Anzeige“ gekennzeichnet. Das Wort „Anzeige“ ist dabei umrahmt (vgl. „Schritt 2“ in Abb. 12.1). Wenn der Nutzer auf eine bezahlte Anzeige klickt, kommt er auf die relevante Landingpage der Webseite, die verlinkt ist. Findet er das passende Produkt und bestellt dieses, löst er eine Conversion aus und der Umsatz wird dem Marketing mit Google AdWords zugeschrieben (Traffic3 o. J.). Abb. 12.1 stellt diesen Ablauf dar. Dass der Kunde ein Produkt kauft und somit eine Conversion auslöst zeigt den Idealfall. Der User könnte die Webseite nach einigen Sekunden oder Minuten auch wieder verlassen, ohne eine Bestellung zu tätigen.

Das Beispiel in Abb. 12.1 basiert auf der Suchanfrage „Kinderfahrrad online kaufen“ und zeigt auf, welche Schritte ein User mindestens durchläuft, bevor er eine Conversion tätigt.

\subsubsection{AdWords Begrifflichkeiten}

Das Verständnis einiger AdWords-Schlagwörter ist essenziell für das Marketing mit Google AdWords. Die Beschreibung der folgenden Begriffe soll helfen, sich einen Überblick über das Themengebiet zu verschaffen.

Impressionen Impressionen bedeuten Einblendungen auf den Google-Ergebnisseiten. Jedes Mal, wenn eine Anzeige bei einer Suchanfrage eines Users erscheint, gilt dies als Impression bzw. Einblendung. Impressionen sind im Suchmaschinenmarketing von Google AdWords kostenlos.

Klicks Klicks entstehen, wenn auf eine Impression auf den Google-Ergebnisseiten geklickt wird. Die Anzahl Klicks gibt an, wie oft Suchende auf ausgespielte Anzeigen geklickt haben. Nach dem Klick landet der User auf der vom Werbenden verlinkten Landingpage. Klicks sind im Suchmaschinenmarketing von Google AdWords kostenpflichtig.

Click through Rate (CTR) Die Click through Rate ist die Kennzahl, welche die Anzahl der Klicks auf Online-Werbeanzeigen im Verhältnis zu der Anzahl der insgesamt erschienenen Impressionen darstellt. Die CTR ist eine wichtige Kennzahl für die Auswertung und Analyse von Google-AdWords-Kampagnen. Gemäß Leu (2018b) wird grundsätzlich eine CTR von $5 \%$ oder höher als erfolgreich angesehen. 


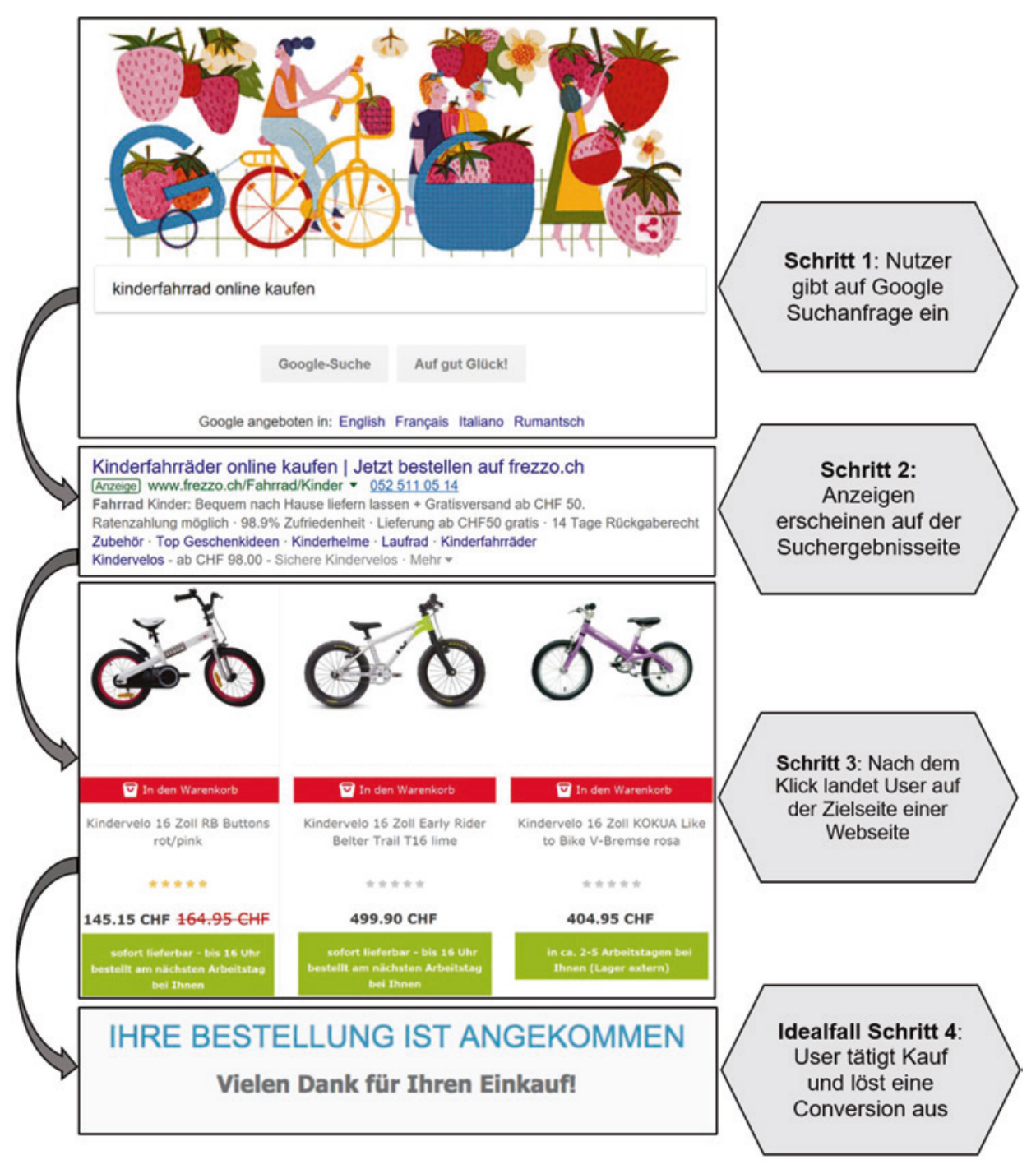

Abb. 12.1 Ablauf einer Google AdWords Conversion. (Eigene Darstellung)

Quality Score Stellt den Qualitätsfaktor für Keywords oder Anzeigen dar. Der Qualitätsfaktor resultiert einerseits aus der Keyword Performance, welche aus den drei Faktoren der historischen Klickrate, der Relevanz für den Anzeigentext sowie der Kontoleistung auf Google besteht. Des Weiteren ist die Webseitenqualität entscheidend, womit Komponenten wie die Mobilgerätefreundlichkeit oder eine einfache Navigation gemeint sind (Leu 2018b). Die Relevanz zwischen Keyword, Anzeige und Landingpage ist eine wichtige Voraussetzung für die Erreichung eines hohen Qualitätsfaktors und beeinflusst 
die Kosten per Klick und den Anzeigerang wesentlich. Der Quality Score kann zwischen 1 und 10 liegen, wobei 1 die schlechteste und 10 die beste Einstufung bedeutet.

Anzeigenrang Der Anzeigenrang resultiert aus der Multiplikation des maximalen CPC(Cost per Click)-Gebots mit dem Quality Score. Das CPC-Gebot entsteht, indem der Werber vorab für jedes Keyword einen maximalen Preis festlegt, den er für einen Klick auf eine seiner Anzeigen zu diesem Keyword zu bezahlen bereit ist (Yapi 2016). Der Anzeigenrang beschreibt die Position, auf welcher die Anzeige auf den Google-Ergebnisseiten eingeblendet wird.

Anzeigenrang $=$ Maximales CPC-Gebot * Quality Score

Auktion Für jede einzelne Suchanfrage gibt es im Hintergrund eine Auktion, in welcher der Google-Algorithmus maßgebend ist und entscheidet, welche Anzeigen eingeblendet werden. Bevor die Auktion extern geht, gibt es vorerst eine interne Auktion, d. h. die eigenen Anzeigen im AdWords-Konto werden gegeneinander geprüft. Pro Suchanfrage geht nämlich jeweils nur ein Keyword mit einer Anzeige ins Rennen, damit der Werber sich nicht selbst konkurrenziert. Das Keyword mit dem höchsten Anzeigenrang wird ins Rennen geschickt (Leu 2018a).

Effektiver CPC Ist der effektive Preis, welcher für einen Klick bezahlt wird. Die Berechnung des effektiven CPC's setzt sich aus der Division des Anzeigenrangs der nachfolgenden Anzeige mit dem eigenen Qualitätsfaktor plus 0,01 CHF zusammen (Leu 2018b).

-Effektiver $\quad \mathrm{CPC}=$ Anzeigenrang nachfolgende Anzeige/eigener Qualitätsfaktor +0,01 CHF

\subsubsection{Kostenmodell}

Die Schaltung von bezahlten Textanzeigen auf der Google Suchmaschine und anderen Suchmaschinen sowie Webseiten, die zum Google-Werbenetzwerk gehören, werden pro Klick auf eine Anzeige abgerechnet, also über einen Klickpreis. Die Werbeform wird auch Pay per Click (PPC) genannt. Dank diesem erfolgsorientierten Kostenmodell zahlt der Werber nur für den Besuch auf der auf einer Anzeige verlinkten Landingpage. Der Klickpreis wird dabei in einem Auktionsverfahren für jedes eingebuchte Keyword und bei jeder Eingabe eines Suchbegriffs von neuem ermittelt (Abschn. 12.3.1.1, „Anzeigenrang“ und „effektiver CPC“) (Yapi 2016). Um zu verhindern, dass ausschließlich diejenigen Anzeigen gute Anzeigepositionen erreichen können, die mit großem Budget gesegnet sind, funktioniert Google AdWords mit der Formel für den Anzeigerang, welche die Relevanz zwischen Keyword, Anzeige und Landingpage miteinbezieht und nicht lediglich das Gebot für den Klick berücksichtigt (Abschn. 12.3.1.1, Anzeigenrang). Google schützt sein Programm damit nicht nur davor, dass ausschließlich Werbende mit hohen Marketingbudgets den Vorrang erhalten und es somit unattraktiv für Unternehmungen mit kleineren Werbebudgets wird, sondern hat auch ein großes Interesse 
daran, seinen Nutzern möglichst relevante Ergebnisse anzuzeigen (traffic3 o. J.). Dies ist eine wichtige Maßnahme, um sicherzustellen, dass die Google User mit den angezeigten Suchergebnissen zufriedengestellt werden.

\subsubsection{Zielsetzung}

Der Inhalt der Webseite, die beworben werden soll, ist Ausgangspunkt für die Festlegung der Werbeziele, die mit Google AdWords verfolgt werden. Eine konkretisierte Zielsetzung ist gemäß der Autorin Dörr von Maydell (2016) wichtig, damit eine Strategie aufgebaut werden kann. Nur so kann die Performance des Marketings mit Google AdWords effektiv ausgewertet werden. Die Zielsetzung soll formuliert werden, bevor das Google-AdWords-Konto eingerichtet wird.

Yapi (2016) betont, dass für die Suchmaschinenwerbung die komplette Marketingplanung (online sowie offline) in Betracht gezogen werden muss. Komponenten wie das Budget, die Laufzeit oder verwendete Formate sollten so weit wie möglich auf andere Werbemaßnahmen abgestimmt sein. Diese Haltung ist damit verknüpft, dass klassische Medien das Interesse für ein Produkt oder eine Dienstleistung wecken sollen. Wer das erweckte Interesse für seine AdWords-Kampagnen nutzen kann, erhöht seine Chancen auf Erfolg. Google unterscheidet im Grundsatz zwischen den drei folgenden Kampagnenzielen.

1. Branding-Kampagne: Steigerung der Markenbekanntheit als Ziel

2. Traffic-Kampagne: Nutzung der Webseiteninhalte als Ziel

3. Direct-Response-Kampagne: Abschließen einer Aktion auf der Webseite als Ziel

\subsubsection{Kontenstruktur}

Ein AdWords Konto besteht aus Kampagnen und Anzeigegruppen, in denen sich die Keywords und Anzeigen befinden. Auf der Ebene des Kontos werden grundsätzliche Komponenten wie Zugriffsrechte, Zeitzone, Währung und Rechnungseinstellungen bestimmt. Auf Kampagnenebene befindet man sich bereits im Detail. Ein weiteres Level darunter befinden sich die Anzeigegruppen, welche die Arbeit mit CPC's für die gesamte Anzeigegruppe, Keywords mit teilweise separaten CPC's, Anzeigentexte und das demografische Targeting beinhalten (Leu 2018a). Auf Kampagnenebene wird zudem der Teil des Google-Werbenetzwerks ausgesucht, in dem die Anzeigen erscheinen sollen. Dabei haben Werbende die Wahl zwischen folgenden Strategien (Yapi 2016):

\section{Suchnetzwerk}

Nach der Eingabe von Begriffen im Google Suchfeld werden über, unter oder neben den organischen Suchergebnissen bezahlte Anzeigen geschaltet (Yapi 2016). Die Voraussetzungen, dass die Anzeigen in den Suchergebnissen erscheinen, sind die Relevanz der Anzeige auf die Suchanfrage sowie ein aktives AdWords-Konto mit einem bestehenden Budget (Learnwithgoolede 2018). Bei der Suchnetzwerkwerbung erscheinen die Anzeigen nur dann, wenn jemand spezifisch und gezielt nach Begriffen 
sucht, welche die werbende Unternehmung in einer Kampagne oder einer Anzeigegruppe als Keyword hinterlegt hat.

\section{Display-Netzwerk}

Anzeigen unterschiedlicher Formate werden auf Webseiten, die entweder ausgewählt werden oder thematisch zu gewissen Keywords passen, platziert. Zudem erscheinen die Anzeigen auf Google-Produkten (Yapi 2016). Mit dem Display-Netzwerk können Werbende ihre Anzeigen auf ausgewählten Websites schalten und dazu auch Fotos oder Videos benutzen. Das Google-Display-Netzwerk umfasst gemäß Learnwithgooglede (2014) über eine Million Webseiten, Smartphone Apps, Videos, Blogs und andere Online-Plattformen, in denen AdWords-Anzeigen erscheinen können. Mit dieser Art von Werbung können über $80 \%$ der globalen Internetnutzer erreicht werden und der Werbende erhält damit eine immense Streumöglichkeit. Im Gegensatz zur Alternative „Suchnetzwerk“ gibt es beim Display-Netzwerk keine vorausgegangene Suchanfrage, welche dazu führt, dass eine relevante Anzeige geschaltet wird. Der Streuverlust ist bei dieser Art von Werbung somit höher.

Werbende können die beiden Werbearten abhängig von den Zielen der AdWords-Kampagnen einzeln benutzen oder kombinieren. Entscheidend ist das Bewusstsein für die Feinstrukturierung des AdWords-Kontos. Je besser die Strukturen gestaltet sind, desto effizienter sind Keywords, Anzeigen und Zielseiten aufeinander abgestimmt. Damit wird nicht nur eine höhere Qualität erreicht, sondern es ist auch möglich, das Budget besser zu kontrollieren (Yapi 2016). Die Kontenstruktur wird gemäß Leu (2018a) im Optimalfall an der Struktur der Webseite ausgerichtet, damit die Abgrenzung der Keyword-Listen und deren Anzeigen simpel getätigt werden kann.

\subsubsection{Keywords, Anzeigegruppen und Anzeigen}

\section{Keywords}

Ein einflussreicher Faktor des Marketings mit Google AdWords sind die Keywords. Die Wahl und Platzierung dieser ist für den Erfolg einer Google-AdWords-Kampagne maßgebend. Denn die Anzeigen erscheinen in den Suchergebnissen nur dann, wenn die Keywords zur Suchanfrage des Nutzers passen. Gemäß Yapi (2016) ist der Ausgangspunkt für eine effiziente Auswahl von Keywords zudem der Inhalt der beworbenen Seite sowie die sprachliche Sichtweise der Besucher.

Bei der Generierung der Keywords ist es zunächst wichtig, Begriffe zu sammeln, die den Inhalt der zu bewerbenden Webseite wiedergeben. Einzahl- und Mehrzahlformen, unterschiedliche Schreibweisen, Synonyme und Worte aus der Alltagssprache sind dabei genauso wichtig wie Fachbegriffe. Grundsätzlich lassen sich Keywords in drei verschiedene Typen unterteilen: 
1. Generische Begriffe: Beschreiben ein Thema, eine Branche oder einen Bereich von Produkten oder Dienstleistungen allgemein, ohne dabei ins Detail zu gehen (z. B. Reisen, Ferien).

2. Spezifische Begriffe: Beschreiben konkrete Unterbereiche, Produkte oder Angebote und sind detaillierter als generische Begriffe (z. B. Städtereise Paris buchen, Ferien Italien All Inclusive).

3. Markenbegriffe: Brand-Keywords bezeichnen ein Unternehmen oder ein Produkt (z. B. Iberostar, Iberostar Hotel Kroatien).

Um zu definieren, welche Suchanfragen die Schaltung einer Anzeige auslösen sollen, bestehen sogenannte Matchtypes, auch Keyword-Optionen genannt (Yapi 2016). Die Funktionsweise dieser ist eine wesentliche Komponente beim Google-AdWords-Marketing. Dem Werbenden stehen fünf Keyword-Optionen zur Auswahl, die unterschiedliche Voraussetzungen für die Ausspielung von Anzeigen bei Suchanfragen des Nutzers aufweisen (Leu 2018b). Die Zuteilung der verschiedenen Keyword-Optionen wird im AdWords-Konto anhand von bestimmten Zeichen ausgeführt. Tab. 12.1 zeigt die Optionen, welche für die Keywords gewählt werden können, inklusive der verwendeten Zeichen.

Tab. 12.1 Übersicht Keyword-Option. (In Anlehnung an Leu 2018b)

\begin{tabular}{l|l|l}
\hline Keyword-Option und Keyword & Anzeige kann erscheinen bei & Anzeige erscheint nicht bei \\
\hline $\begin{array}{l}\text { Weitgehend passend (engl. } \\
\text { bead) }\end{array}$ & $\begin{array}{l}\text { Laptop } \\
\text { Laptop online bestellen } \\
\text { MacBook bestellen } \\
\text { Leistungsstarker PC } \\
\text { Dell Laptop gratis }\end{array}$ & \\
\hline $\begin{array}{l}\text { Weitgehend passend+(engl. } \\
\text { broad match modifier) } \\
\text { Keyword: +Laptop +kaufen }\end{array}$ & $\begin{array}{l}\text { Laptop online kaufen } \\
\text { Laptop kaufen heute } \\
\text { HP Laptop kaufen } \\
\text { Kaufen Laptop }\end{array}$ & $\begin{array}{l}\text { Laptop bestellen } \\
\text { Laptop günstig } \\
\text { Laptop Gewinnspiel }\end{array}$ \\
\hline $\begin{array}{l}\text { Passende Wortgruppe (engl. } \\
\text { phrase) } \\
\text { Keyword: „Laptop kaufen“ }\end{array}$ & $\begin{array}{l}\text { Laptop kaufen heute } \\
\text { Laptop kaufen online } \\
\text { Shop Laptop kaufen }\end{array}$ & $\begin{array}{l}\text { Kaufen Laptop } \\
\text { Laptop online kaufen } \\
\text { Laptop heute kaufen } \\
\text { Laptop günstig }\end{array}$ \\
\hline $\begin{array}{l}\text { Genau passend (engl. exact) } \\
\text { Keyword: [Laptop kaufen] }\end{array}$ & Laptop kaufen & $\begin{array}{l}\text { Laptop bestellen } \\
\text { Jetzt Laptop kaufen } \\
\text { Laptop }\end{array}$ \\
\hline $\begin{array}{l}\text { Ausschließend (engl. negative) } \\
\text { Keyword: -Gebrauchsan- } \\
\text { weisung }\end{array}$ & $\begin{array}{l}\text { Laptop kaufen } \\
\text { Laptop } \\
\text { Günstiger Laptop kaufen }\end{array}$ & $\begin{array}{l}\text { Laptop Gebrauchsanweisung } \\
\text { Gebrauchsanweisung Laptop } \\
\text { Laptop kaufen mit Gebrauchs- } \\
\text { anweisung }\end{array}$ \\
\hline
\end{tabular}


Die fünf Keyword-Optionen funktionieren trichterartig und werden von oben nach unten immer spezifischer. Bei der generischen Option ,weitgehend passend“ sind Anzeigenschaltungen auch bei ähnlichen Wortgruppen oder Synonymen möglich. Diese Option wird für jene Keywords verwendet, die ein geringes Suchvolumen und wenig Markenbegriffe aufweisen (Yapi 2016). Während ein Vorteil dieses Matchtypes darin besteht, dass mehr Impressionen und Klicks generiert werden können, liegen die Nachteile darin, dass die Anzeige möglicherweise für irrelevante Suchanfragen geschaltet wird und somit Klicks entstehen, die nicht nachhaltig sind. Bei dieser Keyword-Option sollen auszuschließende Keywords verwendet und laufend aktualisiert werden (Leu 2018b). Am Ende des Trichters befindet sich der Matchtype „genau passend“, welcher Anzeigen nur dann ausspielt, wenn die Suchanfrage ausschließlich aus dieser Wortgruppe besteht (Yapi 2016). Diese Option erlaubt es, genau zu kontrollieren, für welche Suchanfragen die Anzeigen geschaltet werden. Ausschließende Keywords erübrigen sich bei dieser Option (Leu 2018b). Negative Keywords stellen jene Begriffe dar, zu denen die werbende Unternehmung auf keinen Fall seine Anzeigen schalten möchte.

\section{Anzeigegruppen}

Eine AdWords-Kampagne kann aus einer einzelnen oder mehreren Anzeigegruppen bestehen. Anzeigegruppen sind ein Unterelement der Kampagnen. Es ist pro Keyword auch möglich, in der Anzeigegruppe einzelne maximale CPC-Gebote festzulegen. Mithilfe der Anzeigegruppen können die Anzeigen thematisch sortiert und unterschiedliche Produkte und Dienstleistungen getrennt werden. Die Anzeigegruppen sind ein Strukturelement, um die Keywords und die Anzeigentexte zusammenzufassen. Damit möglichst viele Nutzer angesprochen werden, ist es sinnvoll, für jede Anzeigegruppe mehrere verschiedene Textanzeigen einzupflegen und mit passenden Keywords zu kombinieren (Yapi 2016). Zwei Anzeigen pro Anzeigegruppe sollten mindestens erstellt werden, damit die Erfahrung zeigen kann, welche Anzeige erfolgreicher ist, indem sie gegeneinander getestet werden (Aufgesang GmbH o. J.a).

\section{Anzeigen}

Die AdWords-Anzeigen sind die Werbungen, die den Nutzern auf den Ergebnisseiten angezeigt werden. Die Anzeigen werden in der jeweiligen Anzeigegruppe erstellt und sollten möglichst gut auf die darin enthaltenen Keywords abgestimmt sein. Zusätzlich muss sich die Anzeige auf den Inhalt der beworbenen Seite beziehen, was bedeutet, dass das Angebot des Werbenden die konkreten Vorteile für den Nutzer deutlich darstellen (Yapi 2016). Um eine positive Nutzererfahrung mit den Anzeigen zu erreichen, müssen zwei Kriterien erfüllt sein. Einerseits müssen die Anzeigen relevant, andererseits zutreffend und ehrlich sein. Für die Aufsetzung von Anzeigen bestehen wichtige Richtlinien, die eingehalten werden müssen, damit die Werbeanzeigen ausgespielt werden. Superlativen, die Werbende für sich selbst verwenden, müssen von Dritten bestätigt werden. Das Markenrecht darf nicht verletzt werden und somit ist es nicht erwünscht, 
fremde Marken als Keywords im Anzeigentext zu gebrauchen (Leu 2018b). Die Sorgfalt bei der Erstellung der Textanzeigen ist äußerst wichtig und kontinuierlich zu überprüfen.

Abb. 12.2 zeigt eine Google-AdWords-Werbeanzeige. Die zwei Slogans der ersten Zeile sind die Titelzeilen, die zweite Zeile besteht aus der Anzeige-URL und dem Hinweis, dass es sich um eine bezahlte Werbung (umrahmtes Wort „Anzeige“) hält. Der dritten Zeile kann die Beschreibung des Produkts oder der Dienstleistung entnommen werden.

\subsubsection{AdWords-Vertiefung}

Nachdem Grundbegriffe, wichtige Einzelheiten und die Funktionsweise von Google AdWords im Generellen verstanden sind, bietet eine Vertiefung im Fachgebiet die Chance, sich von der Konkurrenz abzuheben und das Marketing mit Google AdWords zu optimieren. Es bestehen Tools, welche teilweise direkt im AdWords-Konto integriert sind, während andere zusätzlich heruntergeladen werden können.

\subsubsection{Google Analytics}

Google Analytics ist ein Ableger der Unternehmung Google LLC und dient der Datenverkehrsanalyse. Die Haupteigenschaft von Google Analytics besteht demnach aus der Webanalyse und ist ein wichtiges Trackingtool für Unternehmungen, die einen Online-Auftritt haben und diesen optimieren möchten. Das Tool ist eigenständig und somit nicht im Google-AdWords-Konto integriert. Google Analytics erlaubt es, die Entwicklung der Webseite auf einen Blick zu erkennen und die Performance der Webseite zu kontrollieren, optimieren und verbessern.

Gemäß SEO Küche (o. J.a) und Unic (o. J.) sind die Hauptaspekte von Google Analytics folgende:

- Information bezüglich Besucheranzahl, Verweildauer und Verhalten der Besucher.

- Kenntnis der Suchbegriffe, über welche Besucher die Webseite gefunden haben.

- Demografische Merkmale wie Sprache und Standort der Besucher sind bekannt.

- Kenntnis darüber, welche Inhalte der Webseite sich die Besucher angesehen haben.

- Die Devices, welche die Besucher verwenden, sind bekannt.

- Google Analytics hat mehrere bahnbrechende Neuerungen implementiert.

- Die Verknüpfung mit dem Google-AdWords-Konto ist problemlos möglich.

- Die Einbindung von sozialen Netzwerken steht zur Verfügung.

Olivenöl Online Bestellen | Neue Ernte November 2017 Anzeige www.olio-nobile.ch/Bio-Öle -

Handgelesen, direkt vom Baum auf Ihren Tisch. Wir kennen unsere Produzenten.

Abb. 12.2 Grafik einer Werbeanzeige. (Eigene Darstellung) 
- Es können individuelle Ziele definiert und gemessen werden.

- Marketingaktivitäten können gezielt gemessen werden.

Als Nachteil des Tools muss genannt werden, dass Google durch die Einbindung von Google Analytics Zugang zu einer Vielzahl von internen Daten erhält. Es herrscht Unbekanntheit darüber, wie weit Google die gesammelten Daten zur Erstellung von umfassenden Benutzerprofilen nutzt und zukünftig für Marketingzwecke verwenden wird.

\subsubsection{Anzeigenerweiterungen}

Die Anzeigenerweiterungen dienen gemäß Google-AdWords-Schulung (Leu 2018a) einerseits dazu, die Anzeigen optisch größer zu machen und somit mehr Visibilität zu erhalten. Andererseits können die Werbenden die Anzeige mit relevanten Informationen erweitern, um die Attraktivität für den Nutzer zu erhöhen. Überdies sind die Erweiterungen wichtig, um auf den Ergebnisseiten vor der Konkurrenz zu erscheinen. Denn Werbende, welche die Anzeigenerweiterungen nutzen, haben eine erhöhte Chance, einen verbesserten Anzeigenrang zu erreichen, weil seit dem Jahr 2013 die Verwendung von Anzeigenerweiterungen in die Berechnung des Rankings im Google-Algorithmus einfließt und damit die Performance optimieren kann. Google steuert die Inhalte und die Schaltung der Anzeigenerweiterungen Mal für Mal. Es kann auch sein, dass keine Erweiterungen ausgespielt werden, sondern lediglich die bloße, ursprüngliche Anzeige (Yapi 2016). Die Anzeigenerweiterungen sind ein AdWords-internes Tool und der Zugriff läuft direkt in den Kampagnen ab.

Während die klassische Anzeige mit Titel, Anzeige-URL und 80-Zeichen-Beschreibung grundlegende Informationen liefert, geben Anzeigenerweiterungen ausführlichere Inputs bezüglich Produkt, Dienstleistung und/oder Unternehmung. Werbende Unternehmen haben dank den Anzeigenerweiterungen die Möglichkeit, potenziellen Kunden aufzuzeigen, was sie ergänzend zum Produkt oder der Dienstleistung, nach welcher aktuell gerade gesucht wird, noch Zusätzliches zu bieten haben. Zudem beinhalten die Anzeigenerweiterungen sogenannte „Click-to-Call-Buttons“, welche die User dazu aufrufen, eine Aktion zu tätigen, auf eine Anzeige zu klicken, sich den Webseiteninhalt anzuschauen oder Kontakt mit dem Werbenden aufzunehmen (Leu 2018a). In Abschn. „Anzeigenerweiterungen einpflegen“ finden sich Beschreibungen der Anzeigenerweiterungen, welche für das Liveprojekt benutzt wurden. Abb. 12.3 stellt eine Werbeanzeige auf Google AdWords dar, welche mit der Anzeigenerweiterung vom Typ „Sitelink“ ausgestattet ist.

\subsubsection{Search Console}

Die Search Console ist wie Google Analytics ein externes Tool, kann aber ebenfalls mit dem Google-AdWords-Konto verknüpft werden. Bei der Search Console geht es um die grundlegende Thematik, im Web gefunden zu werden. Mithilfe der Search Console können Werbende beobachten, wie die eigene Webseite bei den Suchanfragen abschneidet und anhand davon Optimierungen vornehmen. Mit der Search Console kann 


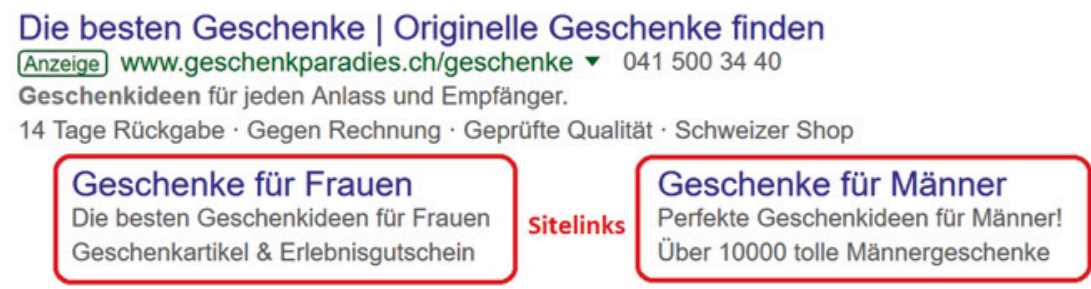

Abb. 12.3 Beispiel für Sitelink-Erweiterungen. (Eigene Darstellung)

sichergestellt werden, dass Google die eigene Webseite auf dem Radar hat und diese den Suchenden bei relevanten Suchanfragen auch einblendet. Ein weiterer Vorteil der Search Console besteht darin, dass das Tool regelmäßig nach Fehlern und Unregelmäßigkeiten sucht und diese meldet, falls negative Aspekte aufgefallen sind. Bei der Erstellung einer neuen Unter- oder Produkteseite, kann dank des Tools nachvollzogen werden, welche Begriffe die Suchenden auf die betroffenen Unterseiten gebracht hat. Somit erhält der Werbende die Möglichkeit, Outperformer zu pushen und diese für andere Unterseiten anzupassen und schlechte Performer zu eliminieren (Google Webmasters 2015). Die Google Search Console ist für die Suchmaschinenoptimierung von großem Wert (SEO Küche o. J.b).

\subsubsection{AdWords-Editor}

Der AdWords-Editor ist ein externes Tool, welches unterstützend für die Arbeit mit Google AdWords eingesetzt und kostenlos heruntergeladen werden kann. Der Editor dient hauptsächlich dazu, große Kampagnen zu bearbeiten. Zudem fungiert das Tool auch als Back-up, denn die Daten müssen vom Editor ins AdWords-Konto kopiert werden. Somit können Tests durchgeführt werden, für welche beispielsweise 100 neue Keywords implementiert und dafür 100 andere Keywords pausiert werden. Nach einiger Zeit können Schlüsse bezüglich Effizienz gezogen und die besser performenden Keywords beibehalten werden. Ein weiterer Vorzug des Tools ist, dass Keywords-Duplikate erkannt und bereinigt werden können (Leu 2018a). Als wichtigste Vorteile des AdWords-Editors können gemäß Google (o. J.) die Aspekte genannt werden, dass das Tool es erlaubt, Änderungen offline vorzunehmen und diese danach ins AdWords-Konto zu übertragen.

\subsubsection{Keyword-Platzhalter}

Dank dem Keyword-Platzhalter kann auf eine hilfreiche Funktion zurückgegriffen werden, welche es möglich macht, das Keyword, welches zur Anzeigenschaltung geführt hat, in einen entsprechenden Platzhalter einfließen zu lassen. Der Keyword-Platzhalter kann für jede beliebige Textanzeige verwendet werden, wobei der Keyword-Platzhalter-Code entweder im Anzeigentitel, in der Beschreibung oder im der angezeigten URL eingebaut wird (Aufgesang GmbH o. J.b). Bei jeder Suchanfrage wird jenes Keyword, welches zur Anzeigenschaltung geführt hat, automatisch in den Platzhalter eingefügt. 
Der Sinn hinter der Idee ist, dass der User in den Suchergebnissen das von ihm eingegebene Keyword eins zu eins in der Anzeige sieht und diese für ihn somit an Relevanz gewinnt. Eine Problematik des Keyword-Platzhalters stellen Falschschreibweisen dar, die in die Anzeige übernommen werden können, falls der Suchende den eingegebenen Begriff falsch buchstabiert. Das Tool ist in Google AdWords integriert und kann bei Bedarf für jede einzelne Anzeige benutzt werden (Leu 2018a).

Die vier beschriebenen Tools sind zwar die bekanntesten und einflussreichsten, sie sind aber nicht abschließend. Google AdWords bietet in weiteren Tools Funktionen und Maßnahmen an, um das Marketing mit Google AdWords zu verbessern. Alle zur Verfügung stehenden Tools oder Funktionen sind darauf ausgelegt, das Marketing mit Google AdWords zu vereinfachen und zu optimieren.

\subsubsection{Optimierungsmöglichkeiten}

$15 \%$ aller täglichen Suchanfragen wurden vorher noch nie genau so verwendet (Leu 2018a). Dieser Fakt verdeutlicht, dass Google AdWords sich in einem äußerst dynamischen Umfeld bewegt. Aufgrund dessen ist es essenziell, dass Werbende am Ball bleiben, mit den Anpassungen, Veränderungen und Neuheiten von Google mitgehen und bestrebt sind, deren AdWords-Konto laufend zu optimieren. Google AdWords bietet dafür Optimierungsmöglichkeiten anhand von verschiedenen Aktionen. Mit diesen Werkzeugen soll es den Werbenden möglich sein, dass wichtige Aspekte nicht vergessen gehen und die Kampagnen komplett sowie ausführlich sind. Ein grundlegender Optimierungstipp von AdWords besagt, dass mit optimalen Anzeigentexten die CTR und somit die gesamte Kampagnenperformance verbessert werden kann. Die Webanalyse ist dabei bei eher tiefen Budgets entscheidend. Beispielsweise kann mit den Webanalysetools die Segmentierung der Präsenz bezogen auf Wochentage oder Tageszeiten ins Auge gefasst und somit starke Wochen- oder Tageszeiten gepusht werden. Erfahrungswerte sind beim Marketing mit Google AdWords äußerst wichtig, da keine allgemeingültigen Aussagen für Kampagnen gemacht werden können und jedes Konto eine eigene Performance mit unterschiedlichen Auffälligkeiten aufweist (Leu 2018a, b).

Um die eigenen Kampagnen ständig zu optimieren und die Detailarbeit so auszuführen, dass das Maximum aus SEA-Maßnahmen herausgeholt werden kann, müssen sich Werbende bei der Auswahl der Keywords des Prozesses zwischen Bewusstsein, Einsatz und Leistung bewusst sein. Damit wird der Online-Kaufprozess vereinfacht abgebildet. Wer diesen Prozess ganzheitlich versteht und seine Keyword-Gruppen sowie Anzeigen daran ausrichtet, kann den potenziellen Kunden leiten und beeinflussen und das Marketing mit Google AdWords dadurch optimieren. Für Werbende ist es von hoher Wichtigkeit, dass die optimale Mischung zwischen generischen und spezifischen Keywords gefunden werden kann und dass sie sich bewusst sind, wonach die Nutzer in den jeweiligen Prozessschritten suchen. Gemäß Google-AdWords-Schulungen (Leu 2018a, b) ist es überdies sinnvoll, den größten Teil des Budgets für spezifische Suchanfragen auszugeben, 
damit die Relevanz der Werbung hoch ist. Gleichzeitig ist es wichtig, dass der ganze Prozess abgedeckt ist und Werbende auch in generische Keywords investieren. Zudem wird zur Optimierung empfohlen, in den Werbeanzeigen mit Saisonalität zu arbeiten. Auch die persönliche Ansprache der Kunden sowie die sogenannten „Call to Action Buttons“ werden als erfolgsversprechend angesehen. Zu guter Letzt wird betont, dass Spezialangebote bestens ankommen, da alle Menschen im Grundsatz Schnäppchenjäger sind.

\subsubsection{Relevanz zwischen Keyword, Anzeige \& Zielseite}

Das meistgenannte Argument für die Erreichung einer guten Performance im Google-AdWords-Konto ist die Relevanz zwischen Keyword, Anzeige und Zielseite. Wenn die eingeblendete Anzeige nicht auf die Suchanfrage des Users passt, wird er nicht darauf klicken, weil die Relevanz nicht gegeben ist. Wenn die Anzeige für die Suchanfrage zwar relevant ist und der Nutzer aus diesem Grund auf die Anzeige klickt, die verlinkte Landingpage am Ende dann aber nicht die gewünschten Informationen bzw. Angebote liefert, ist der User sofort wieder weg. Der Klick hat den Werber dann Geld gekostet, aber der Nutzer wurde nicht befriedigt und dem Werbenden ist es nicht gelungen, gute Werbung zu machen. Diese Entwicklung des Suchanfrageprozesses ist nicht erwünscht. Es ist essenziell, dass die Zielseite genau mit der Anzeige und dem, was darin versprochen wird, übereinstimmt (Leu 2018b). Nebst der Relevanz ist es entscheidend, dass der Inhalt der Landingpage eigenständig und originell ist. Der Text muss authentisch, konsistent und transparent sein. Zudem ist zu beachten, dass die gelieferten Informationen auf der Landingpage prägnant sind und keine langen Textwüsten beinhalten (Leu 2018a). Die Aussage bezüglich Kürze und Prägnanz wird von Oettrich (2016) widerlegt, indem empfohlen wird, lieber mehr als weniger zu schreiben, mindestens aber 300 Wörter. Übergreifend passt der Slogan „Content is King“ ausgezeichnet, wobei für Google entscheidend ist, dass ausreichend Text vorhanden ist, um diesen im Hintergrund durch die Funktionsweise des Google-Algorithmus einzustufen.

\subsection{Methodisches Vorgehen}

In diesem Kapitel werden die empirische Herangehensweise dieses Beitrags detailliert beschrieben und die Instrumente der Erkenntnisgewinnung dargestellt. Auf die Methode der Datenerhebung wird vertieft eingegangen. Zudem wird die Aufsetzung eines Pilotprojekts dargestellt.

Jonker und Pennink (2010, S. 23) empfehlen eine 4-stufige Forschungspyramide als hilfreiches Instrument für die Erarbeitung einer wissenschaftlichen Arbeit. Das Forschungsparadigma, die Forschungsmethodologie, die Forschungsmethode und die Forschungstechnik bilden diese vier Level. Auf allen vier Ebenen werden Entscheidungen getroffen. Die vier Level bestimmen die wissenschaftliche Herangehensweise und die Art der Umsetzung des Praxisprojekts. Die Methodologie dieses Beitrags wird zudem durch die wissenschaftliche Fragestellung beeinflusst. Die Forschungsfrage 
„Welches sind die Erfolgsfaktoren für die Kundengewinnung mit Google AdWords? Ein Pilotprojekt in Zusammenarbeit mit der Hofbauer $\mathrm{GmbH}^{\text {“ }}$ zeichnet sich zum einen durch eine offene Fragestellung aus. Zum anderen zeigt die Forschungsfrage auf, dass ein Pilotprojekt live durchgeführt wird, um die identifizierten Erfolgsfaktoren zu testen. Das Ziel richtet sich demnach deutlich auf die Identifikation von Erfolgsfaktoren beim Marketing mit Google AdWords aus, welche anhand von einer Case Study Analysis aufgedeckt werden sollen. Für die Bestätigung oder Verwerfung der Erfolgsfaktoren dient das Pilotprojekt und für ein fundiertes Vorwissen bezüglich der Themenfelder Online-Marketing und Google AdWords wird eine ausführliche Literaturrecherche vorausgesetzt. Für die Aufdeckung von Erfolgsfaktoren für das Marketing mit Google AdWords eignet sich eine qualitative explorative Erhebung mittels Experteninterviews ausgezeichnet. Dabei wurden einerseits drei Google-AdWords-Experten befragt, um Einblicke in die Welt des Online-Marketings im Generellen und Google AdWords im Spezifischen zu erhalten. Die drei Experten sind bei einem offiziellen Google Partner angestellt, der AdWords-Marketing als Dienstleistung anbietet. Andererseits wurden Experten aus sieben verschiedenen Unternehmen unterschiedlichster Größen und Branchen zu derer Arbeit und Erfahrungen mit Google AdWords interviewt. Die Methodologie ist demnach stark qualitativ geprägt. Diese Herangehensweise wird von Gläser und Laudel (2009, S. 25-29) gestützt. Genanntes Vorgehen erlaubt ein offener Forschungsprozess, was dem noch eher neuartigen und vor allem sehr dynamischen Themengebiet dieses Projekts entspricht.

\subsubsection{Datenerhebung}

Um die qualitative Datenerhebung sorgfältig durchzuführen, orientiert sich die Abwicklung inklusive Vorbereitung und Auswertung an dem von Gläser und Laudel (2010, S. 203) vorgeschlagenen Ablauf. Dieser startet mit theoretischen Vorüberlegungen, welche die Formulierung der Forschungsfrage und die theoretische Analyse und Bestimmung von Variablen beinhaltet, gefolgt von der Extraktions-Vorbereitung, welche die Fixierung des Materials, die Aufnahme von aus der Theorie bekannten Ausprägungen der Variablen sowie der Bestimmung von Indikatoren ins Auge fasst. Der Hauptaspekt dieses Zwischenschritts ist die Festlegung des Samples. Gefolgt wird dies von der Extraktion, welche sich um die Formulierung von Extraktionsregeln, die Aufnahme neuer Ausprägungen, die allfällige Veränderung existierender oder die Konstruktion neuer Variablen und die Aufnahme neuer Indikatoren kümmert. Dieser Schritt wird grob auch Materialdurchlauf genannt und beschreibt die Durchführung der Experteninterviews. Anschließend erfolgen die Aufbereitung der Daten und somit die Sortierung nach zeitlichen oder sachlichen Aspekten, die Zusammenfassung bedeutungsgleicher Informationen sowie die Beseitigung elementarer Fehler. Danach wird die Datenerhebung ausgewertet und dabei fallübergreifende Zusammenhänge und Fälle analysiert (Gläser und Laudel 2010). Nachfolgend wird auf die einzelnen Phasen eingegangen. 


\section{Theoretische Vorüberlegungen}

Die Forschungsfrage „Welches sind die Erfolgsfaktoren für die Kundengewinnung mit Google AdWords? Ein Pilotprojekt in Zusammenarbeit mit der Hofbauer GmbH“ wurde im Rahmen der Vorstudie bestimmt. Ebenso erfolgte in dieser Phase die Erhebung des aktuellen Forschungsstands anhand von Sekundärliteratur. Damit konnten die Forschungslücke sowie die Zielformulierung deutlich abgesteckt werden. Die zur Beantwortung der Forschungsfrage notwendigen Angaben wurden anhand von Experteninterviews sowie der Teilnahme an Google-Kursen eruiert. Damit wurde sichergestellt, dass einzelne Variablen in Abhängigkeit zueinander schließlich die Erfolgsfaktoren des SEA mit Google AdWords identifizieren können. Dank des durchgeführten Pilotprojekts konnten die Variablen geprüft werden. Die qualitative Datenerhebung wurde im Stil einer Case Study durchgeführt.

\section{Vorbereitung Extraktion: Beschreibung des Samples}

Die Literaturrecherche, die Gespräche mit Google AdWords Experten sowie die Teilnahme an Google AdWords Kursen haben aufgezeigt, welche Variablen bereits erhoben wurden und zu welchen Variablen bereits Studien bestehen. Da Online-Marketing und damit einhergehend Google AdWords äußerst aktuelle und populäre Themen sind, bestehen dazu viele Studien und Theorien. Dennoch ist zu betonen, dass keine ganzheitlichen Konzepte für eine erfolgreiche Implementierung eines Google-AdWords-Kontos bestehen.

Bei der Definition des Samples wurde darauf geachtet, dass die Vertreter der Case Study aus verschiedenen Branchen und unterschiedlich großen Unternehmungen stammen, damit möglichst vielfältige Ansichten gewonnen werden können. Zudem wurde das Sample bewusst so erstellt, dass Unternehmungen dabei sind, welche mit Google AdWords ihren Onlineshop bewerben sowie auch Firmen, welche den Fokus nicht auf dem Onlineshop haben oder gar keinen solchen betreiben. Dies weil die Vorstudie ergeben hat, dass die Arbeit mit Google AdWords wesentlich davon abhängt, ob Werbende einen Onlineshop haben und somit direkt Produkte und/oder Dienstleistungen verkaufen oder eben nicht. Um eine zweite Sicht auf das Thema zu erhalten, wurden nebst den Vertretern der Fallstudie drei Google-AdWords-Experten befragt. Aufgrund der unterschiedlichen Problemfelder und Informationsquellen wurden zwei halbstrukturierte Leitfäden erarbeitet, je einen für die Google-AdWords-Experten und einen für die Vertreter der Case Study. Die Leitfäden wurden vorgängig getestet und während der Erhebungsphase laufend angepasst. Außerdem wurden sie kontinuierlich auf dessen Validität geprüft, damit die Resultate zuverlässig erhoben werden konnten. Den Vertretern der Case Study wurde im Vorfeld ein Fragebogen zum biografischen Hintergrund sowie eine auszufüllende Tabelle mit Tools und Eigenschaften von Google AdWords zugestellt, damit eine optimale Vorbereitung auf das Gespräch möglich war und Einblicke in die AdWords-Arbeitsweise der Fallstudienvertreter gewonnen werden konnte. Die Arbeitgeber der Befragten werden aufgrund der Vertraulichkeit der Daten anonymisiert. Der Arbeitgeber der Google-AdWords-Experten wurde umschrieben, 
aber auch anonymisiert. In Tab. 12.2 werden die Google-AdWords-Experten, welche in explorativen Interviews befragt wurden, beschrieben.

In Tab. 12.3 werden die anonymisierten Unternehmungen mit einigen Eckpfeilern beschrieben und die Position des befragten Experten im Unternehmen angegeben. Das Sample besteht aus Unternehmungen aus verschiedenen Regionen der Deutschschweiz.

Tab. 12.2 Interviewpartner - Google-AdWords-Experten. (Eigene Darstellung)

\begin{tabular}{l|l|l}
\hline Name & Arbeitgeber & Position \\
\hline Jeannine Künzler & Offizieller Google Partner, Chur & Leiterin Performance Marketing \\
\hline Tamara Ulber & Offizieller Google Partner, Chur & Medienberaterin \\
\hline Thomas Zimmermann & Offizieller Google Partner, Chur & Online Campaign Manager \\
\hline
\end{tabular}

Tab. 12.3 Interviewpartner - Vertreter Case Study. (Eigene Darstellung)

\begin{tabular}{|c|c|c|}
\hline Name & Unternehmensdetails & Position des Befragten \\
\hline Vertreter Case Study 1 & $\begin{array}{l}\text { Branche: Versicherungen \& Vorsorge } \\
\text { Anzahl MA: }>4000 \\
\text { Gründung: vor } 1850 \\
\text { Jahresumsatz, grob: } 3,5 \text { Mio. CHF }\end{array}$ & Online-Marketing-Manager \\
\hline Vertreter Case Study 2 & $\begin{array}{l}\text { Branche: E-Commerce (Generalist) } \\
\text { Anzahl MA: >40 } \\
\text { Gründung: } 2007 \\
\text { Jahresumsatz, grob: } 28 \text { Mio. CHF }\end{array}$ & Geschäftsführer \\
\hline Vertreter Case Study 3 & $\begin{array}{l}\text { Branche: öffentlicher Verkehr } \\
\text { Anzahl MA: > } 36 \\
\text { Gründung: vor } 1950 \\
\text { Jahresumsatz, grob: } 650.000 \mathrm{CHF}\end{array}$ & $\begin{array}{l}\text { Marketing-Kommunikation, } \\
\text { AdWords-Verantwortlicher }\end{array}$ \\
\hline Vertreter Case Study 4 & $\begin{array}{l}\text { Branche: E-Commerce (Kinderfahr- } \\
\text { zeuge) } \\
\text { Anzahl MA: }<8 \\
\text { Gründung: } 2014 \\
\text { Jahresumsatz, grob: } 1 \text { Mio. CHF }\end{array}$ & Geschäftsführer \\
\hline Vertreter Case Study 5 & $\begin{array}{l}\text { Branche: E-Commerce (Generalist) } \\
\text { Anzahl MA: >100 } \\
\text { Gründung: } 2009 \\
\text { Jahresumsatz, grob: } 42 \text { Mio. CHF }\end{array}$ & AdWords-Verantwortlicher \\
\hline Vertreter Case Study 6 & $\begin{array}{l}\text { Branche: E-Commerce (Generalist) } \\
\text { Anzahl MA: >1000 } \\
\text { Gründung: } 2001 \\
\text { Jahresumsatz, grob: } 830 \text { Mio. CHF }\end{array}$ & $\begin{array}{l}\text { Leiter Digital Marketing \& } \\
\text { Social Media }\end{array}$ \\
\hline Vertreter Case Study 7 & $\begin{array}{l}\text { Branche: Sicherheitstechnik } \\
\text { Anzahl MA: <8 } \\
\text { Gründung: } 2014 \\
\text { Jahresumsatz, grob: } 450.000 \text { CHF }\end{array}$ & Geschäftsführer \\
\hline
\end{tabular}




\section{Extraktion: Durchführung der qualitativen Datenerhebung}

Die Durchführung der Experteninterviews, welche jeweils am Sitz des Unternehmens durchgeführt wurden, dauerte zwischen 40 und 80 min. Während der Extraktion wurden Variablen neu aufgenommen oder verändert und kontinuierlich neue Indikatoren gewonnen. Diese wurden für die darauffolgenden Interviews miteinbezogen.

\section{Aufbereitung}

Um eine systematische und übersichtliche Analyse der qualitativen Datenerhebung sicherzustellen, wurde im Bereich der Experteninterviews mit dem Auswertungstool Atlas.ti gearbeitet. Die Benutzung dieses Tools hat die Erstellung von Codes und Codefamilien ermöglicht, welche aus den Interviewgesprächen extrahiert wurden. Um die Codes zu clustern, wurden Codefamilien gegründet, welche Zitate enthalten, deren verschiedene Codes in einem direkten Zusammenhang zueinanderstehen. Damit eine saubere Codierung gewährleistet werden konnte, wurden alle 10 Interviews in WordDateien transkribiert und die Textdateien mit Zeilennummern versehen, was die exakte Angabe von Quellen vereinfacht. Um die Anonymität der befragten Unternehmen nicht zu gefährden, wurden die Unternehmensnamen in den Transkriptionen mit Codes überschrieben.

\section{Auswertung}

Ein wichtiger Schritt der Auswertung ist die Datenauswertung mittels qualitativer Inhaltsanalyse. Das übergeordnete Ziel dieser Auswertung ist die Erreichung einer Auflistung von Google-AdWords-Erfolgsfaktoren. Um Erfolgsfaktoren aufzudecken, werden die Unternehmungen der Befragten klassifiziert. Als Erfolgs- bzw. Misserfolgsindikator für die befragten Unternehmungen gelten die Zahlen der Umsatzentwicklung des jeweiligen Unternehmens, anhand welcher erklärt werden kann, ob es sich bei der jeweiligen Firma um einen Outperformer oder einen Under-Performer handelt. Die identifizierten Faktoren bei Outperformern geben gemäß der Klassifizierung Anlass, dass es sich um generelle Erfolgsfaktoren beim Marketing mit Google AdWords handelt. Die Interpretation der Ergebnisse aus der Primärforschung erfolgt auch mittels Gegenüberstellung der Erkenntnisse aus der Sekundärforschung, die in der Vorstudie gewonnen werden konnte. Zum Abschluss des Berichts wird der erarbeitete Forschungsstand dargestellt.

\subsubsection{Pilotprojekt}

Aufgrund des starken Praxisbezugs dieses Themenfelds wurde entschieden, ein Liveprojekt durchzuführen, um die gewonnen Erkenntnisse anhand davon zu überprüfen, und damit zu bestätigen oder zu verwerfen. Der Partner für die Implementierung des Pilotprojekts ist ein KMU aus der Region Bern mit der Organisationsform einer GmbH, welches bisher kein Augenmerk auf das SEA mit Google AdWords gesetzt hatte, das 
Potenzial dieses Marketingtools aber als sehr hoch einstuft. Der Partner des Liveprojekts wird in Abschn. 12.6.1 beschrieben.

Die gewonnen Erkenntnisse aus den Gesprächen mit Google-AdWords-Experten, aus Besuchen der beiden Kurse „Learn with Google - Suchmaschinenmarketing mit Google AdWords“ und aus der qualitativen Datenerhebung konnten dank des Pilotprojekts getestet werden. Für die Durchführung des Pilotprojekts wurde vom Geschäftsführer der Partnerfirma ein SEA-Budget von $1000 \mathrm{CHF}$ gesprochen. AdWords arbeitet mit monatlichen Budgets, welche aufgrund der budgetierten $1000 \mathrm{CHF}$ auf $500 \mathrm{CHF}$ pro Monat gesetzt wurde. Da die Kampagnen mit Tagesbudgets ausgestattet werden müssen, wurde das tägliche Budget zwischen $16 \mathrm{CHF}$ und $17 \mathrm{CHF}$ festgelegt und jeweils über die drei bzw. vier laufenden Kampagnen aufgeteilt. Genaueres bezüglich Tagesbudgets und Kampagnen ist in Abschn. 12.6 nachzulesen. Ebenfalls in Abschn. 12.6 finden sich Informationen bezüglich Eröffnung des AdWords-Kontos, Generierung von Keywords, Aufsetzung von Anzeigegruppen sowie Anzeigen und weitere Aufwendungen bezüglich AdWords-Kampagnenerstellung. Die Liveschaltung des Pilotprojekts erfolgte am 6. April 2018 und dauerte knapp zwei Monate. Eine ständige Überwachung und Optimierung sollten in diesem Arbeitsschritt gewährleistet werden. Die Dynamik und Schnelllebigkeit des Online-Marketings stellt eine große Herausforderung in Sachen Aktualität dar. Die laufende Einbringung von gewonnen Erkenntnissen in das Pilotprojekt wurde sichergestellt. Wichtige Einsichten und Meinungen, die aus den durchgeführten Interviews gewonnen werden konnten, wurden kontinuierlich im Pilotprojekt eingebettet. Weitere Kampagnen-Optimierungen wurden mithilfe der Leiterin des Performance-Marketings eines offiziellen Google-Partners aus Chur besprochen und implementiert.

\subsection{Resultate}

Dieses Kapitel rekonstruiert die gewonnen Erkenntnisse aus der qualitativen Datenerhebung mittels Experteninterviews. Das Hauptaugenmerk liegt dabei auf der Darstellung von Erfolgsfaktoren für das Marketing mit Google AdWords.

\subsubsection{Klassifizierung des Samples}

Damit die von den befragten Experten als erfolgsversprechende Aktivitäten für das Marketing mit Google AdWords benannten Aspekte tatsächlich als Erfolgsfaktoren bestätigt werden können, wurde die Umsatzentwicklung der sieben Unternehmungen über die letzten Jahre in Betracht gezogen. Die Unternehmungen wurden anhand dieser Kennzahl sowie dem Einbezug des Gründungsjahrs und der schweizweiten Bekanntheit in die zwei Klassen „Outperformer“ und „moderate Performer“ eingeteilt. Die Datenverfügbarkeit für diese Kennzahl wurde von den interviewten Personen im Nachgang auf Anfrage 
per E-Mail zur Verfügung gestellt. Da die Entwicklung aller befragten Unternehmen als positiv eingestuft werden kann, gilt für diese Forschungsarbeit die Annahme, dass die von den Experten als erfolgsversprechend ausgewiesenen Aspekte tatsächlich Erfolgsfaktoren fürs Marketing mit Google AdWords darstellen. Tab. 12.4 zeigt die Klassifizierung der Arbeitgeber der Interviewpartner.

Die Zahlen 1 bis 7 der Case Study sind für die Arbeitgeber mit der gleichen Ziffer versehen wie in Tab. 12.3. Die Klassifizierung hat ergeben, dass die befragten Unternehmen mit einer Ausnahme durchwegs positive Entwicklungen aufzeigen. Dies beweist, dass die Fallstudie ausnahmslos florierende Unternehmen beinhaltet. Infolgedessen gibt es im Sample keine schwachen Performer, sondern ausschließlich moderate Performer und Outperformer. Die einzige negative Umsatzentwicklung der Unternehmung 3 der Case Study wurde vom befragten Experten relativiert und mit einer prozentualen Steigerung von buchenden Personen (PAX) um 17,5 \% im Jahr 2017 gegenüber 2016 untermauert (Branche von Case Study 3: Öffentlicher Verkehr, vgl. Tab. 12.3).

\subsubsection{Moderate Performer}

Die Arbeitgeber der Vertreter der Case Study mit den Nummern 3, 4, 5 und 7 wurden als moderat erfolgreiche Unternehmen eingestuft. Es ist zu betonen, dass dies keineswegs ein schlechtes Zeugnis ist, denn die Kennzahlen und Entwicklungen der befragten

Tab. 12.4 Klassifizierung des Samples. (Eigene Darstellung)

\begin{tabular}{|c|c|c|}
\hline Unternehmung & Umsatzentwicklung in \% & Klassifizierung \\
\hline Case Study 1 & $\begin{array}{l}2015:+0,72 \% \\
2016:+3,71 \% \\
2017:+3,99 \%\end{array}$ & Outperformer \\
\hline Case Study 2 & $\begin{array}{l}2015:+ \text { ca. } 25 \% \\
2016:+ \text { ca. } 25 \% \\
2017:+ \text { ca. } 25 \%\end{array}$ & Outperformer \\
\hline Case Study 3 & $\begin{array}{l}\text { 2015: keine Angabe } \\
\text { 2016: }+12,75 \% \\
\text { 2017: }-11,16 \%\end{array}$ & Moderate Performer \\
\hline Case Study 4 & $\begin{array}{l}2015:+133 \% \text { (Gründung 2014) } \\
2016:+194 \% \\
2017:+42 \%\end{array}$ & Moderate Performer \\
\hline Case Study 5 & Keine Angaben (Jahresumsatz ca. 42 Mio. CHF) & Moderate Performer \\
\hline Case Study 6 & $\begin{array}{l}\text { 2015: keine Angabe } \\
\text { 2016: }+12,65 \% \\
2017:+18,47 \%\end{array}$ & Outperformer \\
\hline Case Study 7 & $\begin{array}{l}\text { 2015: Gründung } \\
\text { 2016: }+733,3 \% \\
2017:+276 \%\end{array}$ & Moderate Performer \\
\hline
\end{tabular}


Unternehmen lassen sich sehen und sprechen eine hoffnungsvolle Sprache für die Zukunft. Die vier Unternehmungen wurden aufgrund dessen als moderater Performer eingestuft, weil sie zum Teil noch sehr jung sind (Case Study 4 und 7) und die äußerst hohen Umsatzentwicklungszahlen der ersten Jahre noch nicht überbewertet werden dürfen. Die als moderate Performer eingestuften Unternehmungen konnten die prozentuale Umsatzsteigerung von 2015 auf 2016 für das Folgejahr 2017 nicht halten. Die beiden Case Studies 3 und 5 wurden als moderat eingestuft, weil deren Entwicklung gemäß Medien eher stagnieren und auf dem aktuellen Level bestehen bleiben. Wenn diese beiden Unternehmungen ihr Niveau halten können, kann dies jedoch als befriedigend eingestuft werden, denn beide sind im Markt stark etabliert und bewegen sich zudem in einem Marktumfeld, das aktuell nicht stark expandiert.

\subsubsection{Outperformer}

Die Arbeitgeber der Vertreter der Case Study mit den Nummern 1, 2 und 6 wurden als Outperformer eingestuft, was bedeutet, dass sie eine außerordentlich positive Umsatzsteigerung und weitere äußerst nachhaltige Kennzahlen ausweisen können. Zwei der drei klassifizierten Outperformer (Case Studies 1 und 6) sind schweizweit bekannt und dürfen sich selbst in ihren Branchen als Vorzeigeunternehmung betiteln. Es handelt sich dabei um einzigartig erfolgreiche Firmen, die in Wirtschaft und Gesellschaft stark verankert sind. Die Unternehmung hinter Case Study 2 ist jünger, aber sehr dynamisch und ambitiös. Die Firma zeichnet sich seit der Gründung vor gut 10 Jahren durch stetiges und starkes Wachstum (ca. 25 \% Umsatzsteigerung pro Jahr) aus. Die Unternehmen, die als Outperformer eingestuft wurden, konnten ihre prozentuale Umsatzsteigerung über die letzten Jahre halten oder sogar weiter verbessern.

Da alle befragten Unternehmen positive Entwicklungen und Zukunftsaussichten ausweisen, wird in diesem Beitrag davon ausgegangen, dass deren Informationen bezüglich des Marketings mit Google AdWords glaubwürdig und repräsentativ sind. Alle befragten Personen sind ausgewiesene Fachleute im Bereich Online-Marketing und arbeiten seit längerer Zeit, teilweise sogar seit bald einem Jahrzehnt, mit Google AdWords.

\subsubsection{Qualitative Datenauswertung}

Die sieben befragten Personen aus der Fallstudie sowie einer der drei befragten Google-AdWords-Experten wurden im Vorfeld des Interviewgesprächs gebeten, einen Fragebogen zum biografischen Hintergrund, welcher zusätzlich zwei Tabellen bezüglich Arbeitsweisen mit Google AdWords und dessen Tools und Funktionen beinhielten, auszufüllen. Dadurch konnte eruiert werden, wie viele der acht befragten Experten die verschiedenen Tools und Eigenschaften von Google (Abschn. 12.3.2) mit welcher Frequenz benutzen. Auffallend ist dabei, dass sieben Experten angegeben haben, dass sie Anzeigenerweiterungen immer benutzen. Auch die Nutzung von Google Analytics wurde von je vier Befragten mit ,immer“ bzw. „manchmal“ beantwortet. Die 
Auswertung der Fragebögen hat ergeben, dass die Anzeigenerweiterungen sowie Google Analytics eine deutliche Wirkungsweise haben.

Die Analyse der primären Datenforschung hat aufgezeigt, dass durch ein nachhaltiges Marketing mit Google AdWords auch die organischen Suchergebnisse verbessert werden können. Dies stellt damit ein doppelter Vorteil von Google AdWords dar, denn Werbende haben damit die Chance, die Visibilität mit SEA sowie auch SEO zu optimieren (VCS 2 2018 und VCS 3 2018).

\subsubsection{Die Macht von Google AdWords}

Die Auswertung der Datenerhebung hat ergeben, dass ein wichtiger Faktor für die enorm hohe Priorität, welches das Online-Marketing-Tool Google AdWords genießt, vom wichtigen Status der Firma Google gegeben ist. Die Suchmaschine von Google, welche gemäß Lunapark (2017) weltweit einen Marktanteil von $90 \%$ hat, ist ein wichtiges Zugpferd des SEA-Tools Google AdWords. Google stellt jene Plattform zur Verfügung, auf welcher der größte Anteil an Internetusern nach Produkten und Dienstleistungen sucht. Abgesehen davon, ob AdWords ein nutzerfreundliches und ausgereiftes Tool ist, ist es für Werbende wichtig, dort präsent zu sein, wo die Masse sucht, und aus diesem Grund kommen Werbende, Stand heute, nicht an Google und seinen AdWords vorbei, wenn sie SEA betreiben möchten. Dieses Statement wurde von allen 10 befragten Experten abgegeben (Ulber 2018; VCS 1 2018; VCS 2 2018; VCS 3 2018; VCS 4 2018; VCS 5 2018; VCS 6 2018; VCS 7 2018; Zimmermann 2018). Google ist es gelungen, eine einzigartige Suchmaschine mit einem derart ausgereiften Algorithmus zu erschaffen, der schlicht und einfach unersetzbar ist (VCS 1 2018; VCS 4 2018; VCS 7 2018). Der Status einer so klaren, weltweiten Nummer 1 ist aus Werbetreibender Sicht auf der einen Seite sicherlich interessant, da Werbende sich nicht mit mehreren Anbietern auseinandersetzen müssen (VCS 6 2018). Trotzdem muss erwähnt werden, dass ein gewisses Maß an Klumpenrisiko besteht.

\subsubsection{Eigenschaften des Online-Marketings}

Das Nutzerverhalten von Online-Usern ist anders als das Verhalten von Offline-Kunden, und diesen Unterschieden müssen Werbende sich bewusst sein, damit Online-Marketing erfolgreich betrieben werden kann. Gemäß Künzler (2018) benötigt es bei der OnlineWerbung durchschnittlich sieben Kontakte eines Users, bis sich dieser für einen Kauf oder eine andere Aktion (z. B. Ausfüllen eines Kontaktformulars) überzeugen lässt. Es ist für Werbende also wichtig, dass sie Content bieten, dank welchem sich Kunden informieren können. Wenn sie zu einem späteren Zeitpunkt dann bereit sind für den Kauf oder eine Aktion ist die Chance groß, dass sie sich an den Anbieter des Contents erinnern und für die Conversion bzw. den Kauf wieder die Webseite jenes Anbieters aufsuchen (VCS 12018 und VCS 3 2018). Eine andere Eigenschaft des Online-Marketings und seiner Nutzer ist der Fakt, dass das Umfeld sehr schnelllebig und dynamisch ist und alles unmittelbar geschieht (Ulber 2018). Außerdem muss beachtet werden, dass ein Großteil der Kunden hybride Eigenschaften aufweisen. Das heißt es gibt Leute, die Produkte 
oder Dienstleistungen online suchen und sie danach offline im Laden oder Geschäft kaufen und umgekehrt (VCS 1 2018). Die Grundtendenz der Population geht immer mehr dahin, dass sich das Nutzerverhalten stärker auf Online-Tools konzentriert. Die Affinität der Leute für Online-Aktivitäten steigt (VCS 2 2018).

\subsubsection{Chancen des Online-Marketings}

Die Vorzüge des Online-Marketings sind sehr vielfältig. Zusammenfassend setzen sich gemäß Primärforschung folgende Aspekte als entscheidende Vorteile des OnlineMarketings durch:

- Messbarkeit des Erfolgs und damit einhergehend eine saubere Analyse des OnlineMarketings (Ulber 2018; VCS 1 2018; VCS 2 2018; VCS 3 2018; VCS 7 2018; Zimmermann 2018).

- Die Nachvollziehbarkeit von Aktionen dank der Analyse des Nutzerverhaltens ermöglicht die Aufdeckung der Intention von Internetusern. Wenn Werbetreibende es schaffen, sich in den Kunden hineinzuversetzen und Online-Werbung in Bezug darauf zu schalten, kann dies einen sehr wichtigen Wettbewerbsvorteil generieren (Ulber 2018; VCS 1 2018; VCS 3 2018; VCS 6 2018; VCS 7 2018).

- Unmittelbarkeit und Flexibilität, die es ermöglichen, Anpassungen oder Neuheiten sofort zu implementieren und somit kurze Reaktionszeiten aufweisen (VCS 12018 ; VCS 6 2018).

- Zielgerichtete Werbung mit wenig Streuverlust und direkter Ansprache lässt eine Budgetoptimierung zu und sorgt somit für effizientes Online-Marketing (Ulber 2018; VCS 3 2018; VCS 4 2018; VCS 6 2018).

- Die unmittelbare Vergleichbarkeit mit Mitbewerbern und die sofort mögliche Steuerung von verschiedenen Aktionen schaffen Spielräume um sich von den Mitbewerbern abzuheben (VCS 2 2018; VCS 3 2018).

- Im Vergleich zum Offline-Marketing findet im Online-Marketing meistens kein Medienbruch statt, was die Customer Journey optimiert (VCS 6 2018; VCS 7 2018).

Die meistgenannte Chance des Online-Marketings ist die Messbarkeit. Diese ist durch die Einbettung von Trackingcodes möglich, welche aufzeigen, wie ein User sich auf der Webseite verhält, bis er zum Abschluss kommt. Die Abschlüsse oder Käufe werden Conversions genannt und erlauben es, den Erfolg des Online-Marketings zu messen (VCS 1 2018; VCS 3 2018; Zimmermann 2018). Obwohl die Messbarkeit oft als Vorteil des Online-Marketings angeschaut wird, ist die Erfolgsmessung teilweise sehr schwierig, gerade bei Werbetreibenden ohne Onlineshop. Wenn kein Onlineshop vorhanden ist, wo User den Kauf direkt tätigen können, fällt die Messung des Erfolgs schwer. Schließlich kann es sein, dass ein Internetnutzer ein Angebot online gefunden hat, den Abschluss dann aber im Offline-Store vor Ort tätigt. Der Kauf müsste in dem Fall der Online-Werbung zugeschrieben werden. Natürlich ist auch die umgekehrte Option möglich, dass ein Produkt im Offline-Store entdeckt und dann online bestellt wird. Die 
stets korrekte Zuteilung der Werbeeinnahmen ist beinahe unmöglich (VCS 1 2018; VCS 2 2018; Zimmermann 2018). Eine weitere Hürde für die Erfolgsmessung ist die Nichteinbindung von Google Analytics (Abschn. 12.3.2) (VCS 3 2018). Zudem müssen bei der Erfolgsmessung die Werbeausgaben in Betracht gezogen werden, da die simple Betrachtung einer allfälligen Umsatzsteigerung, ohne Kenntnis der Werbekosten, nicht ausreicht. Marge ist dabei das Zauberwort. Diese wird gemäß Marketingexperten (VCS 2 2018; VCS 3 2018; VCS 4 2018; VCS 5 2018; VCS 6 2018) anhand der Kennzahlen KUR, ROI oder ROAS überwacht und optimiert.

\subsubsection{Integrierte Marketingkommunikation}

Die Primärforschung hat aufgezeigt, dass es möglich ist, den Fokus ausschließlich auf einen Marketing-Kanal zu legen (Ulber 2018). Trotzdem machen die Aussagen der Befragten deutlich, dass die Ausführungen von Rumo (2017) bezüglich Einbindung von crossmedialem Marketing im Regelfall die optimale Lösung für werbende Unternehmungen darstellt. Das Angebot eines crossmedialen Marketing-Mixes ist nachhaltig und kann einen Erfolgsfaktor für die Performance einer ganzheitlichen Marketingstrategie darstellen. Mit crossmedialem Marketing ist einerseits gemeint, dass bestenfalls offline sowie online Werbung getätigt wird und andererseits, dass innerhalb der beiden Stränge wiederum verschiedene Kanäle benutzt werden (Ulber 2018; VCS 1 2018; VCS 3 2018; VCS 5 2018; VCS 6 2018; VCS 7 2018; Zimmermann 2018). Im Bereich des Online-Marketings haben sich hauptsächlich Aktivitäten im Remarketing, welche versuchen Leute zurückzuholen, die früher mal auf der Webseite waren, und im Displaynetzwerk, bei welchem Visibilität und somit Brand-Awareness erreicht werden möchte, als effizient herauskristallisiert (VCS 1 2018; VCS 2 2018; VCS 3 2018; VCS 6 2018; VCS 7 2018). Das zur Verfügung stehende Budget spielt beim Implementieren einer integrierten Marketingkommunikation eine zentrale Rolle. Wenn das Budget ausreichend ist, wird der crossmediale Mix unerlässlich, um einen möglichst großen Teil der Leute zu erreichen und somit Kunden zu gewinnen (Ulber 2018; VCS 6 2018; Zimmermann 2018).

\subsubsection{Auffälligkeiten Google AdWords}

Die Datenerhebung hat ergeben, dass die Eintrittsbarrieren beim Marketing mit Google AdWords relativ tief sind. Eine unumgängliche Voraussetzung ist - wie es für Marketingmaßnahmen üblich ist - die Bereitschaft, Budget für eine AdWords-Kampagne zu sprechen (Ulber 2018). Bezogen auf nötige Fachkompetenzen ist die Marketingaktivität mit relativ wenig Vorwissen machbar (VCS 6 2018). Trotzdem ist es von entscheidendem Vorteil, wenn die für AdWords verantwortlichen Personen eine grundlegende technische Affinität aufweisen, analytisch denken und eine Kampagnenperformance auswerten können (VCS 7 2018; Zimmermann 2018). Im großen Ganzen sind AdWords-Kampagnen ein Abbild der Realität, was bedeutet, dass das AdWords-Marketing nur so gut sein kann, wie es die ganzheitliche Leistung der werbenden Unternehmung im Allgemeinen ist (VCS 6 2018). Eine Restriktion von Google AdWords 
liegt in der Bewerbung von neuen, unbekannten Produkten oder Dienstleistungen, die schlichtweg nicht auf dem Netz gesucht und somit auch nicht gefunden werden (VCS 2 2018; VCS 4 2018; VCS 6 2018). Daher macht es keinen Sinn, Werbebudgets für Anzeigen zu sprechen, die Keywords hinterlegt haben, nach denen kein Mensch sucht. Ein Beispiel für einen solchen Fall stellten vor einigen Jahren Laufräder für Kinder dar. Das Produkt hatte sich noch nicht etabliert, das Wort „Laufrad“ war unbekannt und hatte daher kein Suchvolumen (VCS 4 2018).

Funktionsweisen und Eigenheiten des Tools, welche den befragten Experten beim Marketing mit Google AdWords auffallen, sind unter anderem das komplexe Auktionssystem und der damit verbundene stetige Wettbewerb, den aufgrund des äußerst ausgewieften Suchalgorithmus von Google niemand vollständig unter Kontrolle haben kann (VCS 4 2018). Daraus resultiert die Herausforderung, die optimale Strategie zu finden (VCS 2 2018). Die ausgeprägte Dynamik und Schnelllebigkeit des Themenfelds, welche Vergleiche nicht ganz simpel anstellen lässt, sowie die Leitzielsetzung von Google, mit AdWords ein Tool anzubieten, das Internetnutzern relevante Werbung anzeigt, beeinflussen die Funktionsweise von AdWords maßgeblich (VCS 1 2018; VCS 2 2018; VCS 4 2018; VCS 5 2018).

Bezüglich den für Google AdWords ausschlaggebenden Kennzahlen fällt auf, dass die CTR, also das Verhältnis zwischen Impressionen und Klicks, bei Unternehmungen ohne Onlineshop als aussagekräftiger und wichtiger betrachtet wird als bei Werbenden mit Onlineshop. Die befragten Personen der Fallstudie, die mit AdWords hauptsächlich einen Onlineshop bewerben, haben angegeben, dass die Kennzahl der CTR zweitrangig ist. Die Erklärungen dafür lauteten, dass die CTR zwar aufzeigt, ob die geschalteten Anzeigen bei den Usern gut ankommen und gute CTR-Histories in den Auktionsverfahren von Google miteinbezogen werden, Kennzahlen wie die Kosten pro Conversion, also die Kosten pro Abschluss, die Kosten-Umsatz-Relation (KUR) oder der Return on Advertising Spend (ROAS) jedoch bedeutungsvoller sind (VCS 4 2018; VCS 5 2018; VCS 6 2018). Dies hat damit zu tun, dass Werbende mit Onlineshop dank des direkten Kaufs der Kunden eine detailliertere Erhebung von Werbeeinnahmen erhalten und diese dann mit den Werbeausgaben vergleichen können, um die Kampagne als erfolgreich oder weniger erfolgreich einzustufen. Für Produkte oder Dienstleistungen, die nicht mit einem Klick im Internet bestellt werden können, ist es komplizierter, die Einnahmen und damit den Erfolg der Werbung genau zu messen. Daher wird eher auf Kennzahlen wie die CTR fokussiert. Google gibt zur Orientierung vor, dass für eine erfolgreiche Werbung eine CTR von $5 \%$ anzustreben ist (Leu 2018b).

\subsubsection{Chancen des Marketings mit Google AdWords}

Ein Großteil der in Abschn. 12.5.2.3 genannten Chancen des Online-Marketings lässt sich auf Google AdWords übertragen. Dies aus dem simplen Grund, dass AdWords ein Strang im Online-Marketing ist und übergeordnet dieselben Eigenschaften und Vorzüge aufweist wie andere Online-Marketing-Maßnahmen. Diese Aspekte werden in diesem 
Kapitel nicht wiederholt niedergeschrieben. Der Fokus liegt auf spezifischen Vorteilen, welche sich werbende Unternehmungen dank Google AdWords erarbeiten können.

Das Erkennen der Intentionen von Internetnutzern erfährt bei Google AdWords eine vertiefte Ausführung. Aufgrund dessen, dass beim Marketing mit Google AdWords vorausgesetzt wird, dass ein User eine Suchanfrage auf Google startet, bevor die eigene Anzeige geschaltet wird, ist die Relevanz der Werbung höher als beim Gießkannenprinzip. Es werden ausschließlich jene Leute angesprochen, bei denen das Interesse für das beworbene Produkt oder die beworbene Dienstleistung bereits geweckt ist und der Streuverlust daher äußerst gering ausfällt (VCS 6 2018). Man ist im Verkaufsprozess bereits einen Schritt weiter und der Kunde wird bei seinem Interesse abgeholt und begleitet, was innerhalb des Online-Marketings eine einmalige Ausgangslage darstellt (Ulber 2018; VCS 6 2018).

In der Schweiz ist Google AdWords heute teilweise noch in der Findungs- und Implementierungsphase. Das Potenzial zur Ausbaufähigkeit ist in den meisten Regionen groß. Urbane Gebiete wie Zürich oder Bern sind einen langen Schritt weiter als andere Kantone und Regionen (Ulber 2018). Dieser Zustand stellt für Werbende eine Chance dar, da die Konkurrenz überschaubar ist. Andererseits bedeuten im Suchalgorithmus von Google weniger Suchanfragen günstigere Klicks und somit tiefere Kosten für die die werbenden Parteien (Ulber 2018; VCS 7 2018; Zimmermann 2018).

Obschon einige Argumente gegen den Einsatz von Brandkampagnen (Abschn. 12.3.1.3) existieren, hat die Primärforschung ergeben, dass die Bewerbung des eigenen Brands durchaus sinnvoll ist. Ein Grund, sich gegen Brandkampagnen zu entscheiden, ist die Verlinkung der Werbeanzeige mit der Startseite und nicht auf eine spezifische Unterseite. Dies kann die Relevanz der Anzeige negativ beeinträchtigen (VCS 6 2018). Des Weiteren scheinen Brandkampagnen für Unternehmungen, welche in ihrer Branche die Nummer 1 sind, nicht zielführend zu sein (VCS 6 2018). Zu guter Letzt wird bei Verzicht auf Brandkampagnen die zu geringe Bekanntheit des Brands angegeben, was dazu führt, dass keine Suchanfragen dazu stattfinden (VCS 2 2018). Die Argumente, welche für eine Durchführung von Brandkampagnen sprechen, sind dennoch deutlich ausgeprägter. Im Grundsatz ist Visibilität für die Brand-Awareness äußerst wichtig, welche durch Brandkampagnen gewährleistet wird. Zudem wird den Usern Online-Präsenz bewiesen, was eine positive Auswirkung auf den Eindruck der User hat. Brandkampagnen ermöglichen mehr Klicks und im Algorithmus von Google wird die Relevanz der Unternehmen, die Brandkampagnen betreiben, verbessert (VCS 1 2018; VCS 4 2018; Zimmermann 2018). Dies wirkt sich wiederum auf die Auktionen und die bezahlten Klickpreise aus. Als weiterer Schlüsselfaktor für Brandkampagnen gelten die günstigen Klickpreise, welche durch die hohe Relevanz bei der Bewerbung des eigenen Brands entstehen (Ulber 2018; VCS 1 2018; VCS 2 2018; VCS 4 2018; VCS 72018 ; Zimmermann 2018).

Eine weitere Chance des Marketings mit Google AdWords ist die Möglichkeit, für Zielpersonen sehr relevante und zielgerichtete Werbung zu schalten (VCS 1 2018; VCS 3 2018; VCS 4 2018). Zudem ist es dank dem AdWords-Kostenmodell (Abschn. 12.3.1.2) 
mit kleinen Budgets möglich, bei relevanten Suchanfragen Visibilität zu erhalten und gegen die monetäre Übermacht der großen Player auf dem Markt zu konkurrenzieren (Ulber 2018; VCS 2 2018). Weiter besteht bei Google AdWords die Option, sofortige Aktionen bzw. Reaktionen durchzuführen, welche innerhalb von Sekunden angepasst und live geschaltet werden können (VCS 1 2018; VCS 6 2018).

\subsubsection{Google AdWords Erfolgsfaktoren}

In diesem Kapitel werden die wichtigsten Erfolgsfaktoren, die sich bei der Analyse der Datenerhebung herauskristallisiert haben, erklärt. Es ist essenziell, dass Werbende sich nicht ausschließlich auf die Integration der nachfolgenden Erfolgsfaktoren fokussieren, sondern sich den in diesem Kapitel bisher beschrieben Eigenschaften, Chancen und Auffälligkeiten des Online-Marketings sowie von Google AdWords (Abschn. 12.5.2.2, 12.5.2.3 und 12.5.2.5) bewusst sind und diese Faktoren in die Kampagne einfließen lassen.

\subsubsection{Top-Erfolgsfaktor 1: Darstellung und Aufbau der Webseite}

Einer der meistgenannten Faktoren für ein zielführendes Marketing mit Google AdWords ist die Struktur der Webseite, auf welcher die Produkte oder Dienstleistungen beworben werden. Die Qualität der Webseite ist sowohl für Google und die damit verbundene Einstufung der Qualitätsfaktoren als auch für die Internetuser entscheidend, denn diese müssen sich auf der Webseite wohl fühlen und zurechtkommen, ansonsten sind sie sofort wieder weg. In die Struktur der Webseite fließen Faktoren wie die Benutzerfreundlichkeit und der Page Speed ein. Ersteres verlangt eine simple und logische Usability, letzteres ist deshalb wichtig, weil die Nutzer nicht lange Geduld haben und die Webseite sofort verlassen, wenn zu lange Ladezeiten bestehen (Ulber 2018; VCS 1 2018; VCS 7 2018). Die Webseite muss zwingend responsive, d. h. mobile optimiert sein, da ein Großteil der Suchanfragen (ca. $80 \%$ ) über Smartphones statt stationären Desktops gestartet werden (Ulber 2018; Zimmermann 2018). Nebst der Struktur der Webseite ist der darauf präsentierte Inhalt mitentscheidend für die Performance der AdWords-Maßnahmen. Es muss gewährleistet werden, dass die zur Verfügung gestellten Informationen für die User relevant und übersichtlich sind. Mit optimalem Content kann erreicht werden, dass die Leute auf der Webseite verbleiben und nicht gleich wieder abspringen, was im Endeffekt den Qualitätsfaktor erhöht und somit Einfluss nimmt auf den CPC (VCS 2 2018; VCS 3 2018; VCS 4 2018; VCS 5 2018; VCS 6 2018; Zimmermann 2018). Die Webseite fungiert heutzutage als eine Art Visitenkarte, welche einer Unternehmung Glaubwürdigkeit bescheren kann, sofern diese benutzerfreundlich, aktuell und modern daherkommt (Zimmermann 2018).

Äußerungen von interviewten Experten, welche indizieren, dass bei der Werbung mit Google AdWords nicht entscheidend ist, ob der User tatsächlich das beworbene Produkt kauft oder er sich eben für ein anderes entscheidet, beweist, dass die Aufgabe von 
Google AdWords darin besteht, die Leute auf die Webseite des Werbenden zu locken, danach aber der Auftritt der Webseite entscheidend ist für den Erfolg der Kampagnen. Die Customer Journey muss zufriedenstellend sein und diese kann durch die positive Erfahrung auf der Webseite beeinflusst werden (VCS 5 2018; VCS 7 2018). Zusammenfassend kann festgehalten werden, dass der Auftritt und die Gestaltung der Webseite ausgeprägt wichtige Erfolgsfaktoren für das Marketing mit Google AdWords sind.

\subsubsection{Top-Erfolgsfaktor 2: Relevanz zwischen Keyword, Anzeige und Zielseite}

Eng verknüpft mit dem oben dargestellten Erfolgsfaktor ist auch der in diesem Kapitel beschriebene, dank der Datenerhebung identifizierte, Erfolgsfaktor. Es geht im Grundsatz darum, hochrelevante Werbung zu schalten (VCS 3 2018; VCS 7 2018). Für die Erreichung hoher Relevanz beim Marketing mit Google AdWords wird die Dreifaltigkeit zwischen gesuchtem Keyword, geschalteter Anzeige und verlinkter Landingpage erfordert (VCS 1 2018). Die Dreifaltigkeit zwischen Keyword, Anzeige und Landingpage sorgt dafür, dass Internetuser Werbung angezeigt bekommen, die für sie genau passend und somit hochrelevant ist. Dies führt im AdWords-Konto zu einem höheren Qualitätsfaktor (Abschn. 12.3.1.1) und damit zu einer besseren Performance und tieferen Kosten (Ulber 2018; VCS 3 2018; VCS 5 2018; VCS 7 2018; Zimmermann 2018).

Um die Relevanz der Werbung hoch zu halten, ist unter anderem die Verlinkung der korrekten Landingpage in der Anzeige entscheidend. Es ist essenziell, dass Internetnutzer nach dem Klick auf die Anzeige sofort auf der passenden Unterseite der Website landen und relevante Informationen vor sich haben, ohne dass sie sich durch die Webseite klicken müssen (Ulber 2018; VCS 3 2018; VCS 4 2018; VCS 5 2018; VCS 6 2018; VCS 7 2018). Zudem erscheint im Optimalfall das vom User eingegebene Keyword eins zu eins in der ausgespielten Anzeige, um die Relevanz für den potenziellen Kunden zu erhöhen (VCS 6 2018; VCS 7 2018). Ein Instrument, welches dafür sorgt, dass die vom User eingetippte Suchanfrage in der Anzeige ersichtlich ist, ist der Gebrauch des Keyword-Platzhalters (Abschn. 12.3.2) (Zimmermann 2018). Die Primärforschung hat überdies ergeben, dass das Bewerben fremder Brands eine nicht nachhaltige und zu teure Strategie ist. Beim Bewerben von fremden Brands werben Unternehmen mit Namen von sehr bekannten Marken oder Unternehmen (z. B. Digitec Galaxus im E-Commerce oder Zürich Insurance Group in der Versicherungsbranche), um einen Teil der Suchabfragen abzufangen, die spezifisch mit dem Namen des Brands bzw. Players gesucht werden. Dazu buchen Werbetreibende den Brandnamen als Keyword ein, sodass sie bei einer allfälligen Suchanfrage in die Auktion gelangen. Aufgrund dessen, dass mit dieser Strategie Anzeigen ausgespielt werden, die nicht mit der Suchanfrage der User übereinstimmen, ist die Relevanz dieser Anzeigen nicht ausreichend hoch, der Qualitätsfaktor entsprechend niedrig und die Werbekosten daher beträchtlich (VCS 3 2018; VCS 4 2018; VCS 5 2018; VCS 6 2018). 
Google AdWords ermöglicht eine äußerst relevante und spezifische Bewerbung von Produkten oder Dienstleistungen für User, deren Interesse für das Produkt oder die Dienstleistung bereits vorhanden ist. Um eine möglichst hohe Relevanz zu erhalten, ist es wichtig, sich auf die spezifische Schiene der Werbung zu konzentrieren, statt auf die breite Masse abzuzielen (Zimmermann 2018).

\subsubsection{Top Erfolgsfaktor 3: Verknüpfung mit Google Analytics}

Um langfristig von den gespeicherten Daten der AdWords-Aktivitäten zu profitieren und eine saubere, kontinuierliche Analyse zu erhalten, welcher wichtige Informationen zum Nutzerverhalten entnommen werden können, bedarf es der Verknüpfung des AdWords-Kontos mit dem Auswertungstool Google Analytics. Die Datenerhebung hat ergeben, dass Erfahrungswerte der eigenen AdWords-Kampagnen sehr vielsagend sind und tiefgehende Analysen sowie Optimierungspotenzial aufzeigen (VCS 2 2018; VCS 3 2018; VCS 4 2018; VCS 52018 und VCS 7 2018). Obschon oberflächliche Analysen auch im AdWords-Konto herausgezogen werden können, stellt der Erhalt von Zusatzinformationen durch die Einbindung von Google Analytics einen Erfolgsfaktor dar (VCS 2 2018; VCS 5 2018). Unter anderem zeigt Google Analytics detailliert an, welches Verhalten der User nach dem Klick auf die Anzeige an den Tag legt, wie lange er auf der Landingpage der Anzeige und auf der Website im Allgemeinen verweilt (Ulber 2018). Die Kampagnen-Optimierung ist durch Google Analytics zudem möglich, da in Google Analytics Sicht auf das große Ganze gewährt wird und die Daten von Google AdWords nicht separat, sondern in der gesamten Kette des Online-Marketings inklusive Kostendaten aufgezeigt werden (VCS 1 2018; VCS 6 2018). Eine weitere Voraussetzung für eine weiterführende Analyse mit Google Analytics ist das Einbauen von Tracking Codes von AdWords in Analytics (VCS 1 2018), was datenschutztechnisch zwar als problematisch angesehen werden kann. Trotzdem wird die Einbindung von Google Analytics im Generellen und Tracking Codes im Speziellen bei Marketingaktivitäten mit Google AdWords dringend empfohlen.

\subsubsection{Top-Erfolgsfaktor 4: Anzeigenerweiterungen einpflegen}

Ein wichtiger Erfolgsfaktor, welcher in der AdWords-Schulung betont wurde, hat sich mit der Auswertung der Datenerhebung bestätigt. Es handelt sich dabei um die hohe Priorität der Anzeigenerweiterungen (Abschn. 12.3.2.2) (Leu 2018a). Die Nutzung von Anzeigenerweiterungen führt dazu, dass der Werbende seinen potenziellen Kunden sowie auch Google zusätzliche Informationen bietet. Google stuft die Anzeigen anhand davon höher ein und für die User bedeutet dies, dass sie detailliertere Informationen erhalten können und die Relevanz der Anzeige damit erhöht werden kann (VCS 1 2018; VCS 2 2018; VCS 4 2018; VCS 6 2018; Zimmermann 2018). Mit den Anzeigenerweiterungen heben Werbende sich zudem von den Mitbewerbern, welche diese Tools nicht nutzen, ab und können sich somit einen Vorteil erwirtschaften (VCS 2 2018; VCS 6 2018). Des Weiteren vergrößert die Nutzung von Anzeigenerweiterungen die geschalteten Anzeigen, weil die zur Verfügung gestellten Informationen platziert wer- 
den müssen. Dadurch erhalten Werbetreibende mehr Werbeplatz und Visibilität (VCS 5 2018; Zimmermann 2018). Die Anzeigeerweiterungen senden Signale. Eine aktivierte Anruferweiterung z. B. kann Erreichbarkeit signalisieren und Internetnutzer zu einer Handlung auffordern (VCS 3 2018; VCS 4 2018). Zusammenfassend bieten die Anzeigenerweiterungen mit der angebotenen Vielzahl von Erweiterungen eine breite Palette an abwechslungsreichen Optionen, die Werbende für eine größere Anzeige und damit mehr Visibilität sowie für höhere Relevanz und damit einen verbesserten Qualitätsfaktor nutzen können.

\subsubsection{Weitere Erfolgsfaktoren}

Für ein nachhaltiges und effizientes Marketing mit Google AdWords bedarf es nebst den vier Top-Erfolgsfaktoren der Implementierung weiterer Aspekte, um eine ganzheitlich erfolgreiche Kampagne zu erreichen. Zum einen spielen der Qualitätsfaktor und der Anzeigenrang eine zentrale Rolle, denn diese entscheiden, wie viel der Werbende für den Klick bezahlt. Der Qualitätsfaktor ist stark von den beiden Top-Erfolgsfaktoren 1 und 2 abhängig, denn mit einer optimalen Website und relevanten Anzeigen sind wichtige Voraussetzungen für einen hohen Qualitätsfaktor gegeben (Zimmermann 2018). Mit einem hohen Qualitätsfaktor kann eine Budgetoptimierung erreicht werden, weil die Kosten pro Klick sinken (Ulber 2018; VCS 1 2018; VCS 5 2018; VCS 7 2018). Obschon mit exakten und wahrheitsgetreuen Angaben auf der Website und einer hohen Relevanz zwischen Keyword, Anzeige und Landingpage wichtige Beiträge zur Budgetoptimierung geleistet und somit Kosten gesenkt werden können, ist nicht wegzureden, dass ein höheres Werbebudget vielfältigere Optionen bietet (Ulber 2018; VCS 3 2018; VCS 6 2018; VCS 7 2018).

Um AdWords-Kampagnen sauber auswerten zu können ist es wichtig, dass von Anfang an Zielsetzungen getätigt werden. Es muss klar definiert werden, was mit der Kampagne erreicht werden und worauf der Fokus liegen soll (VCS 1 2018; VCS 3 2018; VCS 5 2018; VCS 6 2018). Die Primärforschung hat ergeben, dass Werbende von der Marketingstrategie ableiten sollen, wie sie in welchen Kanälen präsent sein wollen (VCS 3 2018; VCS 6 2018). Zudem ist auch eine deutliche Abgrenzung der verschiedenen Kampagnen innerhalb des AdWords-Kontos wichtig. Damit kann verhindert werden, dass Werbende sich selbst konkurrenzieren (VCS 1 2018). Bezüglich Optimierung und Anpassung von Kampagneninhalten sind sich die befragten Experten einig, dass eigene Erfahrungen gemacht und verschiedene Dinge getestet werden müssen, um langfristig eine verbesserte Performance hinzukriegen (Ulber 2018; VCS 2 2018; VSC 6 2018; VCS 7 2018). Trial \& Error-Analysen sind für die ausgesprochen hohe Komplexität von Google AdWords wichtig und erfolgsversprechend für die laufende Optimierung der Kampagnen (VCS 4 2018). Nebst dem Testen verschiedener Aspekte sprechen sich die Experten dafür aus, kleinere Anpassungen sofort und kontinuierlich anzubringen (VCS 2 2018; VCS 7 2018). Größere, kampagnenübergreifende Optimierungen hingegen sollen erst nach einer Zeitspanne von ca. 2-3 Monaten vorgenommen werden, da sich eingepflegte Aspekte im Google-Algorithmus zuerst 
einpendeln müssen, bevor sie ausgewertet werden können (Ulber 2018; VCS 7 2018; Zimmermann 2018).

\subsubsection{Zwischenfazit}

Nach ausführlicher Gestaltung der Resultate aus der qualitativen Erhebung kann die Forschungsfrage ,Welches sind die Erfolgsfaktoren für die Kundengewinnung mit Google AdWords? Ein Pilotprojekt in Zusammenarbeit mit der Hofbauer GmbH“ umfassend beantwortet werden. Die Experten konnten mit Ausführungen zu ihrer Arbeit und den Erfahrungen beim Marketing mit Google AdWords wichtige Anhaltspunkte darüber liefern, welche Aspekte zwingend beachtet werden müssen, um mit AdWords Erfolg zu haben. Es hat sich herausgestellt, dass Relevanz das bedeutende Stichwort ist für eine effiziente Arbeit mit Google AdWords.

Für den gesamten Auftritt des Online-Marketings ist es unabdingbar, dass die User Experience positiv und zielführend ist. Der Internetnutzer soll sich durch OnlineMarketing-Aktivitäten angesprochen fühlen und zu einer Handlung bewegt werden. Es soll jedoch eine angenehme Erfahrung sein, ohne negativ geprägten Handlungsdruck. Die werbende Unternehmung muss einen guten Eindruck hinterlassen und transparent aufzeigen, was sie anbietet, um sich von der Konkurrenz abzuheben. Dazu ist das Aufzeigen von Unique Selling Propositions (USP), Key Performance Indicators (KPI) und damit einhergehend Wettbewerbsvorteilen grundlegend. Ein konsistentes Gesamtpaket des Online-Auftritts führt dazu, dass der Customer Journey wohlwollend ist und die Kunden damit nachhaltig an die Unternehmung gebunden werden können.

\subsubsection{Handlungsempfehlungen}

Nach Abschluss der Datenanalyse werden folgende Handlungsempfehlungen für das Marketing mit Google AdWords abgegeben.

\section{Top-Erfolgsfaktoren implementieren und pflegen}

Die vier Top-Erfolgsfaktoren (Abschn. 12.5.3.1, 12.5.3.2, 12.5.3.3 und 12.5.3.4) werden mit einigen wenigen Ausnahmen allesamt von allen 10 befragten Experten empfohlen, was die Dringlichkeit dieser Maßnahmen unterstreicht. Aufgrund dessen ist es unentbehrlich, dass Werbende sich ausführlich mit den Erfolgsfaktoren auseinandersetzen und Fokus darauf legen. Wenn diese Aspekte nachhaltig eingepflegt sind, ermöglicht dies optimale Voraussetzungen für eine ausgezeichnete Basis, um mit Google AdWords Erfolg zu haben. Der Schnelllebigkeit dieses Business muss jedoch Rechnung getragen werden, was eine kontinuierliche und anhaltende Optimierung und Anpassung erfordert.

Die Implementierung der vier Top-Erfolgsfaktoren erhöht die Relevanz des Marketings aus Sicht der potenziellen Kunden sowie auch aus Sicht des Anbieters Google LLC, welche beide eine tragende Rolle spielen, wenn es um den Erfolg mit Google AdWords geht. Eine kampagnenübergreifend hohe Relevanz ist als Erfolgsgarant anzuschauen und dazu ist eine Stringenz zwischen Keyword, Anzeige und Landingpage ausschlaggebend. 


\section{Chancen des Online-Marketings sowie von Google AdWords beachten}

Das Marketing in Online-Kanälen unterscheidet sich wesentlich zum Marketing in Offline-Kanälen. Dieser Fakt muss beachtet werden und gerade ein hohes Bewusstsein bezüglich Chancen des Online-Marketings kann einen wichtigen Wettbewerbsvorteil bringen. Einer der auffälligsten Aspekte des Online Marketings ist die Unmittelbarkeit, welche die stetige Aufrechterhaltung des aktuellen Stands erfordert und Marketing in Echtzeit ermöglicht (Abschn. 12.2.2). Kurze Reaktionszeiten in diesem schnelllebigen Umfeld bedeuten einen Vorteil gegenüber der Konkurrenz zu haben und von der Dynamik der Branche profitieren zu können. Dies setzt Flexibilität und Agilität voraus. Sich in die User hineinzuversetzen, um zu erfahren, wie das Nutzerverhalten in spezifischen Situation aussehen könnte und die User dadurch bewusst zu lenken und steuern, hat ebenfalls positive Einflüsse auf das Marketing mit Google AdWords (Abschn. 12.5.2.2 und 12.5.2.3).

\section{Eigenen Weg gehen, Erfahrungen sammeln und davon profitieren}

Jede werbende Unternehmung hat aufgrund des beworbenen Angebots, der Finanzkraft, der Bekanntheit, des Know-hows, der Erfahrung und der Möglichkeiten sehr viele, individuelle Eigenheiten, die sich von allen anderen Mitbewerbern unterscheiden. Dies führt dazu, dass jede AdWords-Kampagne einmalig ist und spezielle Eigenschaften aufweist. Aufgrund dessen ist es essenziell, dass Unternehmungen, welche mit Google AdWords werben wollen, ihre eigenen Erfahrungen sammeln, diese analysieren, auswerten und anhand davon laufende Verbesserungen erzielen können.

\subsection{Pilotprojekt}

In diesem Kapitel werden die Erfahrungen des Liveprojekts dokumentiert. Sie sollen Einblicke in den Aufbau der Kampagne geben, die identifizierten Erfolgsfaktoren - die laufend berücksichtigt wurden - erkennen lassen, Herausforderungen aufzeigen und das Fazit des Liveprojekts darstellen.

\subsubsection{Projektpartner}

Die Hofbauer GmbH mit Sitz in Jegenstorf BE wurde im Jahr 2013 gegründet und bewegt sich in verschiedenen Bereichen der Sportbranche. Einerseits bietet die Unternehmung individuelle Arbeits- und Vereinsbekleidung an, welche nach Wunsch bedruckt und bestickt werden kann. Ein Teil stellt dabei auch das Merchandising dar, indem Vereinsartikel, wie z. B. Minishirts, Baseball Caps, Bodenteppiche etc., individuell gestaltet und bedruckt werden können. Clubs und Vereine gehören damit zu den wichtigsten Zielgruppen. Das zweite Standbein der Firma sind Events und Camps. Da die Gründer der Firma, zwei Brüder, langjährige Unihockey-Nationalspieler sind, geben sie ihre 
Erfahrung und ihr Know-how in Unihockey Camps, Trainings und Trainingstagen an die Jugend und andere Wissbegierige weiter und sorgen so für eine nachhaltige Entwicklung der Sportart Unihockey. Aktuell arbeiten vier Mitarbeitende für die Unternehmung, davon ist jedoch nur der Geschäftsführer in Vollzeit beschäftigt. Der Bekanntheitsgrad der vor knapp sechs Jahren gegründeten Unternehmung hat Steigerungspotenzial. Anhand des Liveprojekts wagte sich die Unternehmung zum ersten Mal an das Thema Google AdWords heran. Dieser Beitrag wertet die Zahlen und Ergebnisse des GoogleAdWords-Marketings der Firma Hofbauer GmbH für eine Zeitspanne von knapp zwei Monaten aus.

\subsubsection{Aufbau Pilotprojekt}

Nachdem die Gespräche mit AdWords Experten, die Teilnahme an Google-Kursen und der Theorieteil dieses Beitrags dazu führten, dass wichtiges Know-how bezüglich Google AdWords gewonnen wurde, wurde die Befragung der Fallstudienvertreter durchgeführt, um das erarbeitete Know-how weiter zu vertiefen. Auf dieser Basis konnte das Pilotprojekt, welches täglich überwacht und optimiert wurde, live geschaltet werden. In diesem Kapitel wird detailliert beschrieben, wie das Google-AdWords-Konto der Firma Hofbauer GmbH aufgebaut wurde.

\subsubsection{Erstellung AdWords Konto \& Kampagnen}

Bevor das Marketing mit Google AdWords live geschaltet werden konnte, wurde ein AdWords-Konto erstellt und bestmöglich vorbereitet. Das Pilotprojekt hat am 6. April 2018 effektiv gestartet und endete am 2. Juni 2018. Somit dauerte es etwas mehr als acht Wochen und weist für die Analyse 58 aktive Tage auf. Bevor das AdWords-Konto eröffnet und strukturiert wurde, wurden an der bestehenden Webseite einige Komponenten angepasst. Beispielsweise mussten die Begriffe für die Menüpunkte optimiert werden, damit diese eine sinnvolle Unterteilung der AdWords-Kampagnen zulassen. Die Begriffe in der Menüleiste lauten seit der Anpassung „Home“, „Bekleidung“, ,Vereinsartikel“ und „Unihockey“, welche die Hauptgeschäftsfelder der Unternehmung darstellen. Zudem wurden allgemeine Informationen wie die Rubrik „Über uns“ ausgebaut, damit den Webseitenbesuchern ausführlichere Angaben über die Firma sowie den Personen dahinter gegeben wird. Nach der Anmeldung des Google Kontos wurden im AdWords-Tool vorerst die verschiedenen Kampagnen definiert und erstellt. Insgesamt wurden vier Kampagnen definiert, welche gemäß Tipp der Google-AdWords-Grundlagenschulung (Leu 2018b) auf die Menüleiste der bereits bestehenden Webseite ausgerichtet wurde (Abschn. 12.3.1.4).

Nebst der Brandkampagne, die „Brand“ genannt wurde, wurden anhand der Menüleiste aus Abb. 12.4 folgende drei Kampagnen aufgesetzt: „Bekleidung“, „Vereinsartikel“ und „Unihockey“. Zusätzlich wurde die Brandkampagne implementiert, da die Datenerhebung ergeben hat, dass diese Art von Kampagnen für die Steigerung des 


\section{h图fbauer}

Home Bekleidung $\vee$ Vereinsartikel $\vee$ Unihockey

Abb. 12.4 Menüleiste der Webseite. (Eigene Darstellung)

Bekanntheitsgrads wichtig ist. Die Kosten dafür halten sich in Grenzen und somit wird für niedrige Klickpreise Visibilität gewährleistet, wenn User nach dem Brand suchen (Abschn. 12.3.1.3 und 12.5.2.6).

Bei der Aufsetzung der vier Kampagnen wurden nachstehende Eigenschaften gewählt, die zu Beginn jeder Kampagnenimplementierung festgelegt werden müssen:

1. Kampagnennamen: „Brand“, „Bekleidung“, „Vereinsartikel“, „Unihockey“

2. Typ: Nur Suchnetzwerk. Der Typ Suchnetzwerk wurde gewählt, damit vorerst ausschließlich Leute angesprochen werden, die aktiv auf Google nach den beworbenen Produkten oder Dienstleistungen suchen und diese effektiv bei Google im Suchfeld eingeben. Somit wurde auf Einblendungen ohne vorausgehende Suchanfragen verzichtet, die ansonsten auf im Voraus bestimmten Webseiten ausgespielt werden (Displaynetzwerk). Hauptgründe dafür waren die begrenzten Ressourcen an Zeit und Geld für das Pilotprojekt.

3. Standorte: Variante „Ich möchte selbst wählen“ ausgewählt. Dann wurden Kantone der Deutschschweiz angewählt. Der Kanton Fribourg wurde miteinbezogen, da er zweisprachig ist und in der Nähe des Unternehmenssitzes der Hofbauer GmbH liegt.

4. Gebotsstrategie: Variante „manueller CPC“ ausgewählt. Damit sind Anpassungen der Gebote für den Cost per Click für jedes Keyword einzeln und manuell möglich.

5. Budget: $17 \mathrm{CHF}$ pro Tag. Google rechnet mit 30 Tagen pro Monat. 30 Tage à $17 \mathrm{CHF}$ ergeben $510 \mathrm{CHF}$ pro Monat, die in dieser Zeitspanne maximal ausgegeben werden durften (Abschn. 12.3.1.2). Das Tagesbudget wurde auf die verschiedenen Kampagnen aufgeteilt. Dieses wurde anhand von Prioritätsangaben des Partners für das Pilotprojekt vergeben. Dabei wurde entschieden, dass 1 CHF pro Tag für die Branding-Kampagne, 8 CHF pro Tag für die Bekleidungskampagne und je 4 CHF pro Tag für die Vereinsartikel- und Unihockey-Kampagne investiert werden soll (Tab. 12.5).

\section{Anzeigengruppen}

Gemäß Leu (2018b) wird sinnvollerweise für jede Produktegruppe eine Anzeigegruppe erstellt und für jede Anzeigegruppe danach eine spezifische Keyword-Liste hinterlegt (Abschn. 12.3.1.5). Dabei sollten für die Einhaltung der Einfachheit und Nutzerfreundlichkeit die Anzeigegruppen an die Unterpunkte der Menüleiste angeglichen werden. Diese Vorgaben wurden eingehalten. 
Tab. 12.5 Strukturierung der Anzeigegruppen. (Eigene Darstellung)

\begin{tabular}{l|l|l}
\hline Kampagne & Anzeigegruppen & Tagesbudget \\
\hline Brand & 1. Hofbauers.ch & $1,00 \mathrm{CHF}$ \\
\hline Bekleidung & $\begin{array}{l}\text { 1. Individuelle Vereinsbekleidung } \\
\text { 2. Freizeit- \& Arbeitsbekleidung } \\
\text { 3. Kollektion Kempa/Uhlsport }\end{array}$ & $8,00 \mathrm{CHF}$ \\
\hline Vereinsartikel & $\begin{array}{l}\text { 1. Duschtïcher } \\
\text { 2. Flexfit-Caps } \\
\text { 3. Minishirts }\end{array}$ & \\
\hline Unihockey & $\begin{array}{l}\text { 1. Camps } \\
\text { 2. Trainingstage }\end{array}$ & $4,00 \mathrm{CHF}$ \\
\hline
\end{tabular}

\section{Anzeigen und Anzeigentexte}

Nachdem die Kampagnen sowie die Anzeigegruppen definiert waren, ging es darum, die Werbeanzeigen zu erstellen. Diese fungieren als Bindeglied zwischen den hinterlegten Keywords und der verlinkten Landingpage der Webseite. Bei der Anzeigenerstellung wurden jeweils folgende Punkte durchlaufen:

1. Anzeigetyp: Textanzeige. Alle anderen Typen wurden in diesem Forschungsprojekt nicht benötigt, da diese über den Kampagnentyp „Nur Suchnetzwerk“ hinausgehen.

2. Finale URL: Für jede Anzeige muss die passende Unterseite der Website verlinkt sein. Es ist wichtig, dass User nicht auf der Home-Seite landen, sondern direkt an relevante Informationen auf der gewünschten Landingpage gelangen (Leu 2018b).

- Beispiel Pilotprojekt: https://www.hofbauers.ch/vereinsbekleidung/wunschdesign/für Anzeigen, welche die Anzeigegruppe „Individuelle Vereinsbekleidung“ betreffen.

3. Erster und zweiter Anzeigentitel: Dafür stehen je 30 Zeichen zur Verfügung. Die beiden Titel werden mit einem Trennstrich separiert und sollen anzeigen, was mit der spezifischen Anzeige angeboten wird.

- Beispiel Pilotprojekt: 1. Titel: Individuelle Vereinsbekleidung, 2. Titel: Turnvereine \& Indoorsportarten

4. Pfad: Stellt die gewünschte URL dar, die in der Anzeige eingeblendet wird. Diese beginnt mit der gewöhnlichen URL der Startseite und kann danach mit $2 \times 15$ Zeichen, getrennt durch ein Slash, nach eigenen Vorstellungen ergänzt werden.

- Beispiel Pilotprojekt: hofbauers.ch/bekleidung/bedrucken

5. Beschreibung: Für die Umschreibung des Angebots stehen maximal 80 Zeichen zur Verfügung. Mit diesen 80 Zeichen erhält der Werbende die Möglichkeit, sein Angebot zu spezifizieren und das Interesse der User zu wecken.

- Beispiel Pilotprojekt: Die beste Adresse für Sublimationsdruck

Abb. 12.5 packt die beschriebenen Komponenten 1 bis 5 in eine Anzeige, welche in abgebildetem Format in den Google Suchergebnissen eingeblendet wird, wenn der Algorithmus errechnet hat, dass die Anzeige ausreichend relevant ist, um ausgespielt zu werden. 
Abb. 12.5 Beispiel-Anzeige. (Eigene Darstellung in Anlehnung an Leu 2018b, S. 65)
Individuelle Vereinsbekleidung - Turnvereine \& Indoorsportarten

Anzeige hofbauers.ch/bekleidung/bedrucken

Die beste Adresse für Sublimationsdruck.

\section{Keywords}

Das Herzstück von Google AdWords sind die in den Anzeigegruppen hinterlegten Keywords. Die Keywords bestehen aus den Angeboten an Produkten und Dienstleistungen, welche die werbende Unternehmung bewerben möchte. Die Auswahl der hinterlegten Keywords entscheidet, ob die Anzeigen eines Werbenden bei einer Suchanfrage eines Nutzers in die interne sowie externe Auktion (Abschn. 12.3.1.1) gehen und somit die Chance haben, eingeblendet und angeklickt zu werden. Vorgängig wurde die Applikation „PPC Keyword Generator“ heruntergeladen. Diese sorgt dafür, dass die bestimmten Keywords auf alle möglichen Weisen kombiniert und somit eine große Anzahl an Keywords generiert wird, die in die jeweils passende Anzeigegruppe eingebettet werden. Insgesamt konnten somit 11.155 Keywords erstellt werden, von denen viele wie erwartet ein geringes Suchvolumen aufweisen und nicht relevant sind. Auf diesem vorerst breit gefächerten Weg können unwichtige Keywords laufend deaktiviert werden und die Möglichkeit besteht, verschiedene Kombinationen zu testen, um zu sehen, welche Keywords am effektivsten sind. Jene Keywords, nach denen nicht gesucht wird, werden nicht ausgespielt und somit auch nicht angeklickt und generieren daher keine Kosten. Dieses Vorgehen wurde von Jeannine Künzler empfohlen, die eine fundierte Expertise mit dem Aufsetzen von AdWords-Kampagnen aufweist. Überdies hat auch die Fallstudienanalyse ergeben, dass Keywords vorerst breit gestreut werden, um danach trichtermäßig Einschränkungen einfließen zu lassen (VCS 1 2018).

\subsubsection{Dokumentation Pilotprojekt}

In diesem Kapitel soll verdeutlicht werden, welche spezifischen Handlungen für das Pilotprojekt durchgeführt wurden, was diese gebracht haben und wie sich die implementierten Erfolgsfaktoren im Liveprojekt eingespielt haben. Die Click through Rate, also das Verhältnis zwischen Einblendungen und Klicks, fungiert im Pilotprojekt als maßgebende Kennzahl für den Erfolg bzw. Misserfolg der AdWords-Kampagne des Pilotprojekts.

\subsubsection{Implementierung der identifizierten Erfolgsfaktoren}

Die Handlungsempfehlungen (Abschn. 12.5.3.7), die sich aus der Fallstudienanalyse sowie den Gesprächen mit AdWords-Experten ergeben haben, wurden laufend integriert. 


\section{Darstellung und Aufbau Website}

Die von Google vergebenen, dynamischen Qualitätsfaktoren hängen stark mit der Qualität von Webseiteninhalten der verlinkten Landingpages zusammen (Abschn. 12.3.1.1 und 12.5.3.1). Um die Qualitätsfaktoren der Keywords zu verbessern, wurden Bemühungen zur Optimierung des Inhalts der verlinkten Unterseiten unternommen. Dabei wurde der in Abschn. 12.3.3.1 beschriebenen optimalen Textlänge von mindestens 300 Wörtern pro Unterseite Beachtung geschenkt. Zusätzlich wurden die hinterlegten Keywords bewusst im Inhalt der Unterseite eingebaut, um die Relevanz der Werbung zu erhöhen. Diese Maßnahmen führten zum Erfolg, welcher anhand der verbesserten Qualitätsfaktoren im Google-AdWords-Konto belegt werden kann. Als Beispiel hierfür wird die Optimierung der verlinkten Unterseite der Anzeigegruppe ,individuelle Vereinsbekleidung“ (Tab. 12.5) aufgeführt. Der Text der Landingpage wurde am 13. April 2018 von 183 auf 475 Wörter erweitert und die im AdWords eingebuchten Keywords wurden bewusst im Text miteinbezogen. Diese Maßnahmen führten dazu, dass sich die Aufteilung der Qualitätsfaktoren von Keywords dieser Anzeigegruppe gemäß Tab. 12.6 veränderte. Die Angabe nach dem Multiplikationszeichen zeigen den erreichten Qualitätsfaktor.

Gemäß Leu (2018c) und VCS 7 (2018) ist es erstrebenswert, Qualitätsfaktoren von 6 oder mehr zu erreichen. Tab. 12.6 zeigt eine deutliche Verbesserung des Verhältnisses der Qualitätsfaktoren im Verlauf der Kampagne auf. Der Page Speed sowie die Auflösung der Bilder auf der Website wurden kontrolliert. Diese Anforderungen sind erfüllt, ebenso wie die Anforderung einer mobile optimierten Webseite. Diese Voraussetzungen sind unterstützend für die Erreichung von hohen Qualitätsfaktoren.

\section{Relevanz zwischen Keyword, Anzeige und Zielseite}

Um die Dreifaltigkeit der Relevanz zwischen Keyword, Anzeige und Zielseite zu erfüllen, wurden im Pilotprojekt verschiedene Maßnahmen ergriffen. Von Beginn weg wurde darauf fokussiert, dass die eingebuchten Keywords mit der aufgesetzten Anzeige stark korrelieren und die Anzeige ausschließlich Informationen bietet, welche sich auf der in der Anzeige verlinkten Zielseite in ausführlicher Form wiederfinden. Dadurch sollte sichergestellt werden, dass die Relevanz der AdWords-Aktivitäten hoch ist.

Ein Indikator für die Relevanz einer Kampagne ist die CTR. Zwar kann diese Kennzahl die Relevanz zwischen Anzeige und der verlinkten Unterseite nur beschränkt

Tab. 12.6 Veränderung des Qualitätsfaktors nach Optimierung der Website. (Eigene Darstellung)

\begin{tabular}{l|l}
\hline Qualitätsfaktoren per 13.04.2018 & Qualitätsfaktoren per 02.06.2018 \\
\hline $0 \times 10 / 10$ & $1 \times 10 / 10$ \\
\hline $1 \times 8 / 10$ & $6 \times 8 / 10$ \\
\hline $1 \times 7 / 10$ & $17 \times 7 / 10$ \\
\hline $19 \times 6 / 10$ & $32 \times 6 / 10$ \\
\hline
\end{tabular}


bestätigen. Dafür zeigt das Verhältnis zwischen Impressionen und Klicks deutlich auf, ob die Relevanz zwischen den eingebuchten Keywords und den ausgespielten Anzeigen gegeben ist. Wenn User auf die ausgespielte Anzeige klicken ist dies ein Indiz dafür, dass ihnen die Anzeige die für sie relevanten Informationen bietet. Gemäß den Aussagen von befragten AdWords-Experten (Ulber 2018; Zimmermann 2018) ist die CTR ab $5 \%$ als sehr gut einzustufen. Das Pilotprojekt erreichte über die Laufzeit von knapp zwei Monaten eine CTR von 9,32\% und übertrifft die Anforderungen für eine Einstufung „CTR ist gut" deutlich. Die gute Performance der CTR ist teilweise auf den Fokus Relevanz zurückzuführen, welcher sich als wichtiger Erfolgsfaktor herauskristallisiert hat und während des Pilotprojekts durchgehend berücksichtigt wurde.

Eine weitere Optimierung ist gemäß Künzler (2018) und VCS 6 (2018) der Ausschluss von negativen Keywords (Tab. 12.1 und Abschn. 12.3.1.5). Dadurch kann verhindert werden, dass bei bestimmten, für den Werbenden irrelevanten Suchanfragen die eigenen Werbeanzeigen ausgespielt und Kosten für Klicks generiert werden. Hauptsächlich bei der Branding Kampagne, welche den Nachnamen Hofbauer beinhaltet, wurden im AdWords regelmäßig die Suchanfragen überwacht, welche zu Klicks geführt hatten. Wenn die Suchanfragen offensichtlich nichts mit der Hofbauer GmbH zu tun hatten, wurden die Wörter als auszuschließendes Keyword erfasst.

\section{Auswertungen mit Google Analytics}

Die Verknüpfung des AdWords-Kontos mit Google Analytics wurde gleichzeitig mit der Implementierung der Kampagne durchgeführt. Die Auswertungen in Google Analytics wurden jedoch aufgrund von Zeitmangel nicht detailliert analysiert und allfällige daraus gewonnene Erkenntnisse daher nicht in das Projekt eingepflegt. Diese Aktivitäten hätten den Rahmen dieses Beitrags gesprengt. Es wird der Hofbauer $\mathrm{GmbH}$ für die zukünftige Arbeit mit Google AdWords jedoch empfohlen, das Nutzerverhalten der Webseitenbesucher vertieft zu analysieren und die eigene Kampagne anhand davon zu optimieren. Nichtsdestotrotz stellt die Verknüpfung des AdWords-Kontos mit Google Analytics einen wichtigen Faktor für das Liveprojekt dar. Es bietet nämlich interessante Informationen zur Performance der Kampagne. Vorerst wurde die Entwicklung der Besucherzahlen auf der Webseite der Hofbauer GmbH angeschaut. Dabei wird der Zeitraum zwischen dem Kampagnenstart am 6. April 2018 bis zum Ende des Pilotprojekts am 2. Juni 2018 mit dem gleichen Zeitraum unmittelbar davor verglichen. Diese Analyse hat ergeben, dass dank des Google-AdWords-Projekts für die Hofbauer GmbH eine Steigerung von 21,8 \% auf 1476 Webseitenbesucher in der Zeitspanne des Projekts erzielt werden konnte. Diese Zahl darf als äußerst positiv gewertet werden, da zusätzlicher Traffic auf der Website von gut einem Fünftel eine beträchtliche Anzahl an potenziellen Neukunden mit sich bringt.

\section{Anzeigenerweiterungen einpflegen}

Aufgrund der hohen Wichtigkeit der Nutzung von Anzeigenerweiterungen, welche sich während der Datenerhebung herauskristallisiert hat (Abschn. 12.5.3.4), wurde klar, dass 
die Erweiterungen für das Liveprojekt genutzt und ausprobiert werden müssen. Der Fokus wurde auf die Anzeigenerweiterungen, genannt Snippet-, Sitelink- \& Anruferweiterung, sowie der Erweiterung mit Zusatzinformationen gelegt. Letztere werden auch Callouts genannt und finden sich in der geschalteten Anzeige als zusätzliche Informationen zum Unternehmen. Nachfolgend werden die Performances der genutzten Anzeigenerweiterungen während des Pilotprojekts dargestellt:

Callouts sind Erweiterungen mit Zusatzinformationen und finden sich in der geschalteten Anzeige als zusätzliche Informationen zum Unternehmen. Im Liveprojekt ist auffällig, dass die CTR aller Callouts über $10 \%$ und somit über dem durchschnittlichen CTR des Projekts (9,32\%) liegen.

Die Snippets, welche ähnlich wie die Callouts als Zusatzinformationen in der Anzeige fungieren sind im Gegensatz zu den Callouts mit zur Verfügung stehenden Kategorien vorgegeben. Für das Pilotprojekt wurden die Kategorien „Marken“ und „Dienstleistungen“" auserwählt und das Angebot der Hofbauer GmbH anhand davon beschrieben. Die Snippet-Erweiterungen liegen mit einer durchschnittlicher CTR von 10,14\% ebenfalls über der CTR des gesamten Projekts (9,32\%).

Kleine, separate Anzeigen innerhalb der geschalteten Anzeige werden Sitelinks genannt (vgl. Abb. 12.3). Die Sitelinks zeigen den Usern Angebote an, welche das werbende Unternehmen nebst dem aktuell gesuchten Produkt oder der Dienstleistung zusätzlich anbietet. Sitelinks sorgen zudem dafür, dass die Anzeige größer wird und mehr Werbeplatz einnimmt. Auch die Sitelink-Erweiterungen weisen im Liveprojekt mit einer durchschnittlicher CTR von 10,43\% einen überdurchschnittlichen Wert im Vergleich zur CTR des gesamten Liveprojekts $(9,32 \%)$ auf.

Die vierte genutzte Erweiterung ist die Anruferweiterung, bei der über die geschalteten Anzeigen die Telefonnummer der Hofbauer GmbH angezeigt wird und auf die bei der Suche mit einem Smartphone geklickt werden kann, um den Anruf direkt einzuleiten. Die Performance der Anruferweiterung mit 1095 Einblendungen und 124 dazugehörigen Klicks ergibt eine CTR von 11,32\%, was im Allgemeinen einen starken und fürs Pilotprojekt höheren Wert als die durchschnittlichen 9,32\% darstellt.

Die bei allen implementierten Anzeigenerweiterungen höheren CTR als die durchschnittliche CTR von 9,32\% geben Aufschluss darüber, dass die Anzeigen erfolgreicher waren, wenn sie mit den in den einzelnen Kampagnen eingebetteten Erweiterungen ausgespielt wurden, als wenn sie in der Anzeige nicht ersichtlich waren. Daraus wird geschlossen, dass die Relevanz der Anzeigen bei Ausspielung der Anzeigeerweiterungen höher ist als bei Nichteinblendung der Anzeigenerweiterungen. Abschn. 12.5.3.4 hält fest, dass Werbende sich mit der Nutzung von Anzeigenerweiterungen von der Konkurrenz abheben und sich somit einen Wettbewerbsvorteil verschaffen können. Die Kennzahlen der Anzeigenerweiterungen für das Pilotprojekt lassen den Schluss zu, dass die Performance dank der Implementierung von Callouts, Snippets, Sitelinks und der Anruferweiterung verbessert wurde. Der Top-Erfolgsfaktor 4 (Abschn. 12.5.3.4) kann somit durch das Liveprojekt bestätigt werden. 


\subsection{Diskussion und Fazit}

Google AdWords bietet eine effiziente Plattform für Online-Marketing. Der Beitrag hat aufgedeckt, dass die Relevanz, welche sich wie ein roter Faden durch die Marketingmaßnahmen ziehen soll, das magische Wort beim Marketing mit Google AdWords ist, um eine positive Auswertung der Ergebnisse zu erlangen. Gemäß Erkenntnissen der Datenerhebung und dank der Durchführung des Pilotprojekts kann die folgende Hypothese gewagt werden: effizientes Marketing mit Google AdWords erfordert als Basis ein attraktives Angebot an Produkten und/oder Dienstleistungen, eine moderne und nutzerfreundliche Webseite, eine sorgfältige Stringenz zwischen Werbung und Angebot und somit eine hohe Relevanz zwischen eingebuchten Keywords, geschalteten Anzeigen und verlinkten Zielseiten sowie eine fortlaufende Überwachung und Optimierung des Google-AdWords-Kontos mit Einbezug von spezifischen Eigenheiten, welche jede einzelne werbende Unternehmung aufweist, und Erfahrungen, die im Laufe der Zeit gewonnen werden.

Die Datenerhebung hat ergeben, dass die Schnelllebigkeit und Dynamik des Themenfelds Online-Marketing und Google AdWords (Abschn. 12.5.2.5) sowie die individuellen Eigenheiten und Funktionsweisen der werbenden Unternehmungen dazu führen, dass es kein allgemeingültiges Rezept für erfolgreiche AdWords-Strategien gibt. Die Einhaltung der Top-Erfolgsfaktoren (Abschn. 12.5.3) bietet jedoch eine wertvolle Grundlage für effizientes Google-AdWords-Marketing.

Die Auswertung des Liveprojekts bestätigt die anhand der Datenerhebung aufgedeckten Erfolgsfaktoren. Obschon keine detaillierte Beschreibung der Zielsetzung für das Pilotprojekt ausgearbeitet wurde und somit eine saubere Auswertung des Liveprojekts erschwert wird, darf die AdWords-Kampagne der Hofbauer $\mathrm{GmbH}$ als erfolgreich gewertet werden. Von den in Abschn. 12.3.1.3 dargestellten Kampagnentypen können ausschließlich die zwei Typen Branding- sowie Traffic-Kampagnen für die Hofbauer GmbH relevant sein, da der dritte Typ, Direct-Response-Kampagnen, auf Conversions und somit Abschlüsse zielen. Deren Messung ist nur für Werbende mit Onlineshop, die Verkäufe sofort eins zu eins nachvollziehen können, sinnvoll. Die Branding-Kampagne hat eine Steigerung der Markenbekanntheit als Ziel, was für die junge Partnerfirma des Liveprojekts einen wichtigen Aspekt darstellt (Abschn. 12.6.1). Für Branding-Kampagnen gelten Impressionen als Messwert für den Erfolg. Während der AdWords-Kampagne wurden die Werbeanzeigen des Pilotprojekts 7040 Mal eingeblendet, was einem Tagesdurchschnitt von 120 Impressionen entspricht. Obwohl unbekannt bleibt, wie viele User die Anzeigen tatsächlich wahrgenommen bzw. angeschaut haben darf festgehalten werden, dass der Brand der Unternehmung gegen außen getragen und von der Öffentlichkeit wahrgenommen wurde.

Das Pilotprojekt liegt mit einer durchschnittlichen CTR von 9,32\% deutlich über der CTR von $5 \%$, welche als Grenzwert für die Einstufung als ,gute Performance“ gilt. Der zweite Kampagnentyp, welcher für Unternehmen ohne Onlineshop messbar ist, 
sogenannte Traffic-Kampagnen (Abschn. 12.3.1.3), haben die Nutzung von Webseiteninhalten als Ziel und deren Erfolg misst sich an Klicks auf die Werbeanzeigen, die auf eine Zielseite der Webseite führen. Durch die Livekampagne haben 656 Klicks auf die Website der Partnerfirma geführt und die Besucherzahl (direkte, organische und bezahlte Zugriffe) ist um 21,8\% im Vergleich zur gleichen, vorangehenden Zeitspanne auf 1476 Nutzer angestiegen. Dank des erhöhten Traffic verfügt die Hofbauer GmbH heute über eine deutlich höhere Anzahl an potenziellen Kunden. Die Aussage des Geschäftsführers der Hofbauer $\mathrm{GmbH}$ darüber, dass Kontakt- und Offertanfragen seit Projektstart zugenommen haben, deckt sich mit dem Aspekt, dass während des Pilotprojekts sechs Conversions gemessen wurden. Dabei haben User, welche über AdWords auf die Webseite gelangt sind, unmittelbar nach dem Klick auf die Werbeanzeige das Kontaktformular auf der Webseite ausgefüllt. Aktionen, bei denen User über die bezahlte Suche auf die Webseite gelangt sind, danach aber eine E-Mail über den herkömmlichen Weg gesendet haben oder später über den direkten oder organischen Zugriff auf die Seite zurückgekommen sind und eine Aktion getätigt haben, werden im AdWords nicht als Conversion gemessen, was eine Schwierigkeit der Messbarkeit des Online-Marketings darstellt (Abschn. 12.5.2.3).

Dieser Beitrag bestätigt, dass Google AdWords ein effizientes Tool des Online Marketings darstellt und vielen Unternehmen eine geeignete und sinnvoll einsetzbare Plattform bietet.

\section{Literatur}

Advidera. (о. J.). Real time advertising. https://www.advidera.com/glossar/real-timeadvertising/. Zugegriffen: 14. Mai 2018.

Aufgesang GmbH. (o. J.a). AdWords Anzeigengruppe Definition. https://www.semdeutschland.de/ adwords-agentur/adwords-glossar/adwords-anzeigengruppe-definition/. Zugegriffen: 16. Mai 2018.

Aufgesang GmbH. (o. J.b). Dynamic Keyword Insertion (Keyword-Platzhalter) Definition \& Info. https://www.sem-deutschland.de/adwords-agentur/adwords-glossar/dynamic-keyword-platzhalter/. Zugegriffen: 17. Mai 2018.

Beck, A. (2011). Google AdWords (3. Aufl.) Heidelberg: eine Verlagsgruppe der Hüthig Jehle Rehm GmbH.

Bundesamt für Statistik (BFS). (2017). Internetnutzung. https://www.bfs.admin.ch/bfs/de/home/ statistiken/kultur-medien-informationsgesellschaftsport/informationsgesellschaft/gesamtindikatoren/haushaltebevoelkerung/internetnutzung.html. Zugegriffen: 14. Mai 2018.

Busch, O. (Hrsg.). (2014). Realtime Advertising. Digitales Marketing in Echtzeit: Strategien, Konzepte und Perspektiven. Wiesbaden: Springer Gabler.

Dänzler, S., \& Heun, T. (2014). Marke und digitale Medien. Der Wandel des Markenkonzepts im 21. Jahrhundert. Wiesbaden: Springer Gabler.

DIM Deutsches Institut für Marketing GmbH, (o. J.). Google AdWords Seminar, Köln. https:// www.marketinginstitut.biz/seminare/online-marketing/google-adwords-seminar/. Zugegriffen: 29. Dez. 2017.

Dörr von Maydell, H. (2016). Google AdWords-Tipp: Setzen Sie sich Ziele. https://www.heise-regioconcept.de/google/google-adwords-ziele. Zugegriffen: 14. Mai 2018. 
Engel, D. (2015). Ist Realtime die Echte Zeit? Springer Professional. 2015. https://www.springerprofessional.de/marketing-+-vertrieb/markenstrategie/ist-realtime-dieechte-zeit/6599162. Zugegriffen: 14. Mai 2018.

Europäisches Parlament. (2018). Online-Shopping für alle: Ende von Geoblocking. http:// www.europarl.europa.eu/news/de/headlines/economy/20180126STO94142/onlineshopping-fur-alle-ende-von-geoblocking. Zugegriffen: 13. Mai 2018.

Frost, S. (2013). Datenschutz ist größtes Hindernis. https://www.tagesspiegel.de/wirtschaft/nonliner-atlas-2013-datenschutz-ist-groessteshindernis/8105722.html. Zugegriffen: 14. Mai 2018.

Gläser, J., \& Laudel, G. (2009). Experteninterviews und qualitative Inhaltsanalyse. Als Instrumente rekonstruierender Untersuchungen (3. Aufl.). Wiesbaden: VS Verlag.

Gläser, J., \& Laudel, G. (2010). Experteninterviews und qualitative Inhaltsanalyse. Als Instrumente rekonstruierender Untersuchungen (4. Aufl.). Wiesbaden: VS Verlag.

Google. (o. J.). AdWords Editor Hilfe. https://support.google.com/adwords/editor/answer/2484521?hl=de\&ref_topic $=3290839$. Zugegriffen: 17. Mai 2018.

Google AdWords. (o. J.). Schalten Sie Anzeigen bei Google. http://www.google.ch/adwords/?channel=ha\&subid=ch-de-ha-aw-aw-cb1_xx_txx_xx_xx_bau-non!o2 118682095-138815685732kwd-19087281652. Zugegriffen: 30. Dez. 2017.

Google Webmasters. (2015). Google search console. https://www.youtube.com/watch?v=SoxU5kz15Kc. Zugegriffen: 17. Mai 2018.

GoogleWatchBlog. (2015). 15 Jahre AdWords: Infografik zeigt Statistiken rund um Googles Gelddruckmaschine. https://www.googlewatchblog.de/2015/10/jahre-adwordsinfografik-statistiken/. Zugegriffen: 11. Mai 2018.

Griesbaum, J. (2012). Online marketing. https://www.unihildesheim.de/media/fb3/informationswissenschaft/IIM_IW/griesbaum/GdPIuD_C4.pdf. Zugegriffen: 13. Mai 2018.

Hamburg, I. (2017). Jeder Zweite hat Angst vor Cyberkriminalität. https://www.marketing-boerse. de/News/details/1719-Jeder-Zweite-hat-Angst-vor-Cyberkriminalitaet/138539. Zugegriffen: 14. Mai 2018.

Herrmann, S. (2017). Werbemarkt-Studie von Zenith: Werbung im Social Web überholt Print. https:// www.wuv.de/medien/werbung_im_social_web_ueberholt_print. Zugegriffen: 12. Febr. 2019.

Hohlstein, K. (2016). Die Bedeutung des Online Marketings wächst. https://www.billomat.com/ magazin/die-bedeutung-des-online-marketings-waechst/\#. Zugegriffen: 13. Mai 2018.

Internet World Business. (2018). Diese Player dominieren den weltweiten digitalen Werbemarkt. https://www.internetworld.de/onlinemarketing/google/playerdominieren-weltweiten-digitalenwerbemarkt-1466688.html. Zugegriffen: 12. Febr. 2019.

Jonker, J., \& Pennink, B. W. (2010). The essence of research methodology. A concise guide for master and PhD students in management science. Berlin: Springer.

Learnwithgooglede. (2014). Was ist das Google Display-Netzwerk? https://www.youtube.com/ watch?time_continue $=20 \& v=d r Y Z b 2 g U p E 0$. Zugegriffen: 16. Mai 2018.

Learnwithgooglede. (2018). Einstieg in Google AdWords: Was sind Suchnetzwerk-Anzeigen? https://www.youtube.com/watch?v=t6Uj_eDQpbs. Zugegriffen: 16. Mai 2018.

Leu, E. (2018a). Skript zum Modul "Learn with Google: Suchmaschinenmarketing mit Google AdWords - Vertiefung». Zürich: Google Switzerland.

Leu, E. (2018b). Skript zum Modul "Learn with Google: Suchmaschinenmarketing mit Google AdWords - Grundlagen». Zürich: Google Switzerland.

Leu, E. (2018c). Skript zum Modul «Learn with Google: Suchmaschinenmarketing mit Google AdWords - Vertiefung». Zürich: Google Switzerland.

Link, J. (2000). Wettbewerbsvorteile durch Online Marketing. Die strategischen Perspektiven elektronischer Märkte (2. Aufl.). Berlin: Springer.

Lunapark. (2017). Suchmaschinenmarktanteile weltweit 2017. https://www.lunapark.de/ blog/9907-suchmaschinen-marktanteile-weltweit-2014/. Zugegriffen: 19. Mai 2018. 
McCarthy, E. J. (1960). Basic Marketing. A Managerial Approach. Homewood: Indiana University \& Richard D. Irwin, Inc.

Michnew, E. (2015). Suchmaschinenmarketing abseits von Google AdWords. https://www.amazeemetrics.com/de/blog/suchmaschinenmarketing-abseits-von-googleadwords\#. Zugegriffen: 14. Mai 2018.

Oettrich, S. (2016). 7 Tipps, wie Ihr KMU ein Top-Ranking auf Google erhält. https://www.bag.ch/ blog/27360-2268/7_Tipps_wie_Ihr_KMU_ein_Top-Ranking_auf_Google_erhaelt.html?s=58. Zugegriffen: 20. Mai 2018.

Rumo, E. (2017). Kommunikationsmanagement und Social Media Marketingkommunikation. Skript zum Modul RSSM. Bern: Berner Fachhochschule.

SEO Küche. (o. J.a). Was ist Google analytics? https://www.seokueche.de/lexikon/google-analytics/. Zugegriffen: 16. Mai 2018.

SEO Küche. (o. J.b). Was sind die Google Webmaster Tools? https://www.seo-kueche.de/lexikon/ google-webmaster-tools/. Zugegriffen: 17. Mai 2018.

Stich, K. (2017). Mit dem crossmedialen Marketing Online- und Offline-Marketing verbinden. http://cmm-magazine.ch/mit-dem-crossmedialen-marketing-online-undoffline-marketing-verbinden/. Zugegriffen: 14. Mai 2018.

Stone, M. (2014). Share of global adspend, by medium, 2012 and 2015. https://newsbizblog. org/2014/07/18/share-of-global-adspend-by-medium-2012-and-2015/. Zugegriffen: 14. Mai 2018.

Studer, L. (2008). Studie: Google AdWords und ihr Branding-Effekt, Zürich: Yourposition AG. https://www.yourposition.ch/de/blog/studie-google-adwords-und-ihr-brandingeffekt. Zugegriffen: 2. Jan. 2018.

Traffic3. (о. J.). Wie funktionieren AdWods? https://traffic3.net/wissen/adwords/adwords-ablauf. Zugegriffen: 15. Mai 2018.

Ulber, T. [Medienberaterin] (2018). Identifikation von Erfolgsfaktoren für die Kundengewinnung mit Google AdWords, Interview mit Seraina Ulber vom 23.03.2018.

Unic. (o. J.) Webanalyse: Besser gratis? - Warum für ein Tool zahlen, wenn es Google Analytics gibt? https://www.unic.com/de/kompetenzen/expertenblog/2014/webanalyse-besser-gratis-warum-fuer-ein-tool-zahlen-wenn-es-googleanalytics-gibt. Zugegriffen: 16. Mai 2018.

Vertreter Case Study (VCS) 1 [Online Marketing Manager Unternehmen 1]. (2018). Identifikation von Erfolgsfaktoren für die Kundengewinnung mit Google AdWords, Interview mit Seraina Ulber vom 27.03.2018.

Vertreter Case Study (VCS) 2 [Geschäftsführer Unternehmen 2]. (2018). Identifikation von Erfolgsfaktoren für die Kundengewinnung mit Google AdWords, Interview mit Seraina Ulber vom 28.03.2018.

Vertreter Case Study (VCS) 3 [Marketing-Kommunikation, Google AdWords Verantwortlicher Unternehmen 3]. (2018). Identifikation von Erfolgsfaktoren für die Kundengewinnung mit Google AdWords, Interview mit Seraina Ulber vom 29.03.2018.

Vertreter Case Study (VCS) 4 [Geschäftsführer Unternehmen 4]. (2018). Identifikation von Erfolgsfaktoren für die Kundengewinnung mit Google AdWords, Interview mit Seraina Ulber vom 03.04.2018.

Vertreter Case Study (VCS) 5 [Google AdWords Verantwortlicher Unternehmen 5]. (2018). Identifikation von Erfolgsfaktoren für die Kundengewinnung mit Google AdWords, Interview mit Seraina Ulber vom 03.04.2018.

Vertreter Case Study (VCS) 6 [Leiter digital Marketing \& social Media Unternehmen 6]. (2018). Identifikation von Erfolgsfaktoren für die Kundengewinnung mit Google AdWords, Interview mit Seraina Ulber vom 04.04.2018.

Vertreter Case Study (VCS) 7 [Geschäftsführer Unternehmen 7]. (2018). Identifikation von Erfolgsfaktoren für die Kundengewinnung mit Google AdWords, Interview mit Seraina Ulber vom 06.04.2018. 
Weller, B., \& Calcott, L. (2012). The definitive guide to Google AdWords. Create versatile and powerful marketing and advertising campaigns. New York: Springer.

Wendeler, V. (o. J.). Digitales Marketing - Eine neue Ära für Wachstum. Köln: Wendeler Marketing Consulting. https://www.wemarcon.de/de/marketing/digitales-marketing/. Zugegriffen: 30. Dez.r 2017.

Yapi, L. (2016). Die ganze Welt des Online-Marketings. Google AdWords, Zürich: Swiss Marketing.

Zimmermann, T. [Online Campaign Manager] (2018). Identifikation von Erfolgsfaktoren für die Kundengewinnung mit Google AdWords, Interview mit Seraina Ulber vom 23.03.2018.

Ulber, Seraina (M.Sc. BA/seraina.ulber@bluemail.ch) Masterstudium in Betriebswirtschaft an der Berner Fachhochschule mit Vertiefung Corporate / Business Development. Langjährige Tätigkeit als Kundenbetreuerin im Aussendienst bei Optitrade \& Service AG, einem Importeur und Distributor, der den Schweizer Gross- und Fachhandel beliefert. Ab Juni 2019 bei der Firma Coldtec AG als Key Account Managerin angestellt.

Rumo, Etienne J. (Dr./etienne.rumo@hefr.ch) ist assoziierter Professor für Marketing und Sales Management an der Hochschule für Wirtschaft in Freiburg und Dozent am konsekutiven Masterstudiengang der Berner Fachhochschule mit Studien (MBA) an der University of Edinburgh (UK) und $(\mathrm{PhD})$ der Université de Lorraine (France). Seine Forschungsaktivitäten konzentrieren sich auf Sales Management und International Entrepreneurship. Seit über 10 Jahren begleitet er als Berater KMU's und Grossbetriebe in den Bereichen Verkauf, Vertrieb und International Business Development. 
Open Access Dieses Kapitel wird unter der Creative Commons Namensnennung 4.0 International Lizenz (http://creativecommons.org/licenses/by/4.0/deed.de) veröffentlicht, welche die Nutzung, Vervielfältigung, Bearbeitung, Verbreitung und Wiedergabe in jeglichem Medium und Format erlaubt, sofern Sie den/die ursprünglichen Autor(en) und die Quelle ordnungsgemäß nennen, einen Link zur Creative Commons Lizenz beifügen und angeben, ob Änderungen vorgenommen wurden.

Die in diesem Kapitel enthaltenen Bilder und sonstiges Drittmaterial unterliegen ebenfalls der genannten Creative Commons Lizenz, sofern sich aus der Abbildungslegende nichts anderes ergibt. Sofern das betreffende Material nicht unter der genannten Creative Commons Lizenz steht und die betreffende Handlung nicht nach gesetzlichen Vorschriften erlaubt ist, ist für die oben aufgeführten Weiterverwendungen des Materials die Einwilligung des jeweiligen Rechteinhabers einzuholen.

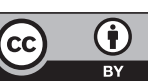

